\title{
Color scrambles reveal Red and Green half-wave linear mechanisms plus a mechanism selective for low chromatic contrast
}

\author{
Christian Herrera Ortiz†, Charles Chubb $\ddagger$, \\ Charles E. Wright $\ddagger$, Peng Sun $\ddagger$, George Sperling $\ddagger$ \\ $\dagger$ Auditory Research Lab, VA Loma Linda Healthcare System, Loma Linda, CA \\ $\ddagger$ Department of Cognitive Sciences, University of California, Irvine, CA \\ July 29, 2021
}




\begin{abstract}
This paper introduces a new method to determine how subjects make discriminations among red-green texture stimuli. More specifically, the method determines (1) the number of mechanisms in human vision sensitive to lights that vary along the constant$S$ cardinal axis (cSCA) of DKL space and (2) the sensitivity of each mechanism to cSCA lights. Each of five subjects was tested in four, separately-blocked tasks. In each task, the subject strove to detect the location of a patch of cSCA-scramble (a spatially random mixture of cSCA lights) in a large, annular background of cSCA-scramble with a different histogram. In different tasks the target patch was (1) redder, (2) greener, (3) higher in red-green contrast, and (4) lower in red-green contrast than the background. For each subject in each task, we measure how target salience is influenced by different cSCA lights. By assuming that in each task each subject uses a weighted sum of his-or-her available mechanisms to construct a "tool" that is optimal for detecting the target, we can derive the sensitivity functions of the mechanisms underlying performance. Results suggest that human vision possesses three mechanisms sensitive to cSCA lights: a red half-wave linear mechanism, a complementary green half-wave linear mechanism, and a third mechanism that is activated by color-scrambles with low chromatic contrast in high-chromatic-contrast backgrounds.
\end{abstract}

Histogram contrast analysis Texture discrimination Color mechanisms 


\section{Introduction}

We will use the term "mechanism" throughout this paper to refer to a cognitively impenetrable process that operates automatically, continuously in time, to transform the image projecting to the retina into a corresponding, neural output image. In more practical terms, the mechanisms initially considered here consist of a color filter that selectively admits a range of wavelengths and an integrator that sums the admitted light to produce a positive output. More complex mechanisms are considered later in Section 4.3.

What color-selective mechanisms exist in human visual perception? This question has been the focus of a great deal of research effort, and yet no clear consensus has emerged.

It is clear, however, that several important visual processes rely on a particular mechanism that extracts the pattern of light intensity impinging on the retina. The term "heterochromatic photometry" refers to the various methods that have been developed for deriving lights of different colors that produce equal activation (called "luminance") in this mechanism. Heterochromatic flicker photometry [Ives, 1912a,b,c,d,e, 1914, 1917, Bone and Landrum, 2004, Walsh, 1958], for example, exploits the fact that rapid temporal alternation of two lights of different color will typically evoke a strong percept of flicker; however, it is possible to adjust the intensity of one of the two lights to sharply attenuate this sense of flicker. This method assumes that the system that senses this flicker takes luminance as its input and that the two lights are equal in luminance at the point of minimum flicker. Similarly, two abutting regions of different color will typically form a clear, easily localized border. However, it is possible to adjust the intensity of one of the lights so that the border weakens and becomes difficult to localize. The "minimally distinct border" method [Kaiser, 1971, Kaiser et al., 1990] assumes that, like the system used to sense flicker, the border-localization system takes luminance as its input. Thus, when the lights are adjusted to make their border as weak as possible, their luminance is equal. Similar logic applies to the minimum-motion method used to obtain the luminance axis of color space for each subject individually in the current study (Sec. 2.3.1).

Substantial evidence suggests that luminance depends very little on $S$-cone activation [Lange, 1958, Eisner and MacLeod, 1980, Guth et al., 1968, Tansley and Boynton, 1978, Tansley and Glushko, 1978, Verdon and Adams, 1987]. (There is, however, compelling evidence that $S$-cone activation does exert a slight negative effect on luminance under conditions of intense, long-wavelength adaptation [Ripamonti et al., 2009].) This observation led MacLeod and Boynton [1978] to propose that the chromaticities of lights should be coded by triples $(s, m, l)$, where $m$ and $l$ code the proportion of the light's luminance contributed by $M$ - and $L$-cones, respectively (thus, $m+l=1$ ), and $s$ reflects the strength of $S$-cone activation relative to the luminance of the light. The key idea here was to dissociate chromatic variation from luminance variation by considering the relative position of a light within the equiluminant plane-the plane of lights all of which have the same luminance as the given light.

This idea was taken up as a central feature of the coding scheme introduced by Derrington et al. [1984] who represented their stimuli in a color space whose axes were (1) luminance, (2) a constant- $S$ axis (called the "constant $B$ " axis in Derrington et al. [1984]) along which chromaticity varies without changing either luminance or $S$-cone activation and (3) a constant- $M-\&-L$ axis (called the "constant $R \& G$ " axis in Derrington et al. [1984]) 
along which chromaticity varies without altering any of luminance or $M$-cone or $L$-cone activation. Insofar as it is true that luminance is invariant with respect to $S$-cone activation, the constant- $M-\&-L$ axis is identical to the gradient of $S$-cone activation (which follows the tritanopic confusion line).

Lights along the constant-S axis vary from reddish to greenish. However, this axis deviates strongly away from the unique red-green axis. The greens tend toward greenish-blue and the reds toward magenta. These are the colors used in the current study. This axis of color space is notable because variations along this axis are not detected by mechanisms whose sensitivity is restricted to either or both of luminance and/or $S$-cone activation. Assuming that luminance reflects the sum of $M$ - and $L$-cone activation, only those mechanisms that are sensitive to differences between $L$ - and $M$-cone activation will respond differentially to variations along the constant- $S$ axis.

\subsection{Half-wave linear mechanisms}

Krauskopf et al. [1982] showed that there exists at least one mechanism that can be fatigued by habituating to a $1 \mathrm{~Hz}$ red-green modulation. That this mechanism was selective for variations in the difference between $L$ - and $M$-activation was suggested by the fact that habituating to this modulation did not affect sensitivity to pulses away from white in the plane spanned by the achromatic axis and the tritanopic confusion line (i.e., the gradient of $S$-cone activation). Thus, whatever mechanisms were used to sense these pulses were not significantly exercised by the red-green modulation.

It is tempting to think of the mechanism whose sensitivity was suppressed by this redgreen modulation as taking a simple difference between $M$-cone and $L$-cone activation. However, substantial evidence suggests that this is not true. Eskew [2009] emphasizes that we should think about the system isolated by red-green habituation as two, complementary, half-wave rectified mechanisms:

- A red half-wave linear mechanism whose response is given by

$$
[L-M]^{+}=\left\{\begin{array}{cc}
L-M & \text { if } L-M>0 \\
0 & \text { otherwise }
\end{array}\right.
$$

- And a green half-wave linear mechanism whose response is given by

$$
[L-M]^{-}=\left\{\begin{array}{cc}
|L-M| & \text { if } L-M<0 \\
0 & \text { otherwise }
\end{array}\right.
$$

The argument supporting this contention involves two assumptions that are intrinsic to the idea of a mechanism: the univariance assumption and the labeled line assumption:

- The univariance assumption holds that the output produced by a mechanism in response to a light is a single number: the activation produced in the mechanism by the light [Rushton, 1972]

- The labeled line assumption [Graham, 1989, Watson and Robson, 1981] holds that two lights will appear different if and only if they produce significantly different levels of activation in some mechanism. 
Suppose, for example, that $B$ is the spectrum of a background light. Let $\delta_{1}$ and $\delta_{2}$ be functions mapping the visible wavelengths into $\mathbb{R}$, and consider a stimulus consisting of a dot with spectrum $B+\alpha_{1} \delta_{1}$ next to a dot with spectrum $B+\alpha_{2} \delta_{2}$ on an otherwise homogeneous background with spectrum $B$. Suppose all mechanisms except one, that we shall call $Q$, are blind to variations in the amplitude of each of $\delta_{1}$ and $\delta_{2}$. In this case, for any values of $\alpha_{1}$ and $\alpha_{2}$, the response of any mechanism other than $Q$ to the lights $B+\alpha_{1} \delta_{1}$ and $B+\alpha_{2} \delta_{2}$ is equal to its response to $B$. Suppose, however, that $Q$ is sensitive (in different degrees) to both $\delta_{1}$ and $\delta_{2}$. The univariance assumption requires that for any $\alpha_{1}$ there exists a value of $\alpha_{2}$ such that the spots with spectra $B+\alpha_{1} \delta_{1}$ and $B+\alpha_{2} \delta_{2}$ appear identical. In particular, this will be true for $\alpha_{2}$ adjusted so that the two spots produce equal activation in $Q$. On the other hand, the labeled line assumption implies that if there exists a mechanism, $Q^{\prime}$ distinct from $Q$ that is sensitive to variations in the amplitude of $\delta_{1}$ but not to variations in the amplitude of $\delta_{2}$, then no matter how one adjusts the amplitude $\alpha_{2}$, there will always be a perceptual difference between the lights with spectra $B+\alpha_{1} \delta_{1}$ and $B+\alpha_{2} \delta_{2}$ because these two lights produce different levels of activation in $Q^{\prime}$.

Eskew [2009] observes that if the background light with spectrum $B$ is gray, and the dots with spectra $B+\alpha_{1} \delta_{1}$ and $B+\alpha_{2} \delta_{2}$ are red and green respectively, then even if the amplitudes $\alpha_{1}$ and $\alpha_{2}$ are both at detection threshold (under additional conditions designed to insure that performance is mediated by a single mechanism), the two dots appear different [Eskew et al., 2001, Krauskopf et al., 1986, Mullen and Kulikowski, 1990]. This leads Eskew to conclude that human vision possesses distinct mechanisms activated by $\delta_{1}$ and $\delta_{2}$. He refers to such mechanisms as half-wave linear mechanisms.

The proposal that there exist separate red and green half-wave linear mechanisms is bolstered by a number of other results. First, adapting to temporal modulations of chromaticity that follow a sawtooth profile (e.g., repeatedly changing gradually to red, then changing abruptly to green and changing gradually again to red, etc.) can increase thresholds differentially for the gradually changing color vs. the color receiving the abrupt onset [Krauskopf et al., 1982, Krauskopf and Zaidi, 1986]. It has also been shown that briefly flashed reddish targets are much more effectively masked by reddish than by greenish noise, and vice versa [Sankeralli and Mullen, 1997, 2001]. In addition, it has been shown that targets that deviate from a neutral adapting field in the red direction vs. those that deviate in the green direction are detected by different mechanisms at threshold in the fovea [Gowdy et al., 1999a,b]. Additional evidence comes from experiments measuring sensitivity to targets that deviate from a neutral adapting field in the red and green directions at different retinal eccentricities. The mechanism that senses green deviations is activated by increases in $M$-cone contrast coupled with equal decreases in $L$-cone contrast; the mechanism that senses red deviations is oppositely tuned. However, as retinal eccentricity increases, sensitivity to green deviations declines faster than sensitivity to red deviations. Several studies have exploited this dissociation in mechanism sensitivity to argue that green and red deviations are sensed by distinct mechanisms [Stromeyer et al., 1992, Newton and Eskew, 2003]. That these mechanisms are post-receptoral is implied by the fact that the $M$ - and $L$-cones contribute with equal strength in both mechanisms.

The current study uses a different experimental paradigm involving discrimination of chromatic textures to seek evidence of red- vs. green-selective half-wave linear mechanisms. This paradigm was originally used by Silva and Chubb [2014] to analyze the mechanisms 
sensitive to textures composed of different grayscales. In the current application, we use textures composed of colors drawn from the constant- $S$ of DKL-space [Derrington et al., 1984]. The textures we use appear similar to some of those used by Li and Lennie [1997]. In that study, however, textures were used in a completely different way than in the current study. Li and Lennie [1997] used textures in which colors were always drawn from a Gaussian distribution along one or another line in color space. The questions of interest in that paper were focused on how the orientation of the distribution-line affected sensitivity to the deviation of the distribution mean from the white point.

The general strategy of the current study, which is sketched in Victor et al. [2017], involves testing subjects in several different tasks, each of which requires the subject to detect the location of a patch of one sort of color texture in a background of a different sort of color texture. The predominant quality of the target patch relative to the background varies across the different tasks. We assume that to perform a given task the subject constructs a taskspecific "tool" by taking a linear combination of his-or-her available mechanisms. We further assume that the weights with which the mechanisms are combined are chosen to maximize the expected difference in activation produced by the target patch versus the background

texture. As we shall demonstrate, under these assumptions, the resulting data enables us to determine

1. that three mechanisms exist in human vision that are differentially sensitive to the colors used in our textures, and

2. the sensitivity of each of these mechanisms to the different colors.

\section{Methods}

\subsection{Subjects}

There were 5 subjects ( 2 female) one of which was the first author. Subjects (other than the first author) were naive to the purpose of the experiments. All reported normal or corrected-to-normal vision. None were color-deficient. The UC Irvine Institutional Review Board approved the experimental procedures, and all subjects provided signed consent.

\subsection{Equipment}

An iMac desktop computer running OS X version 10.6.8 with a $3.06 \mathrm{GHz}$ Intel Core 2 Duo processor and $4 \mathrm{~GB}$ memory capacity was used for stimuli presentation and data collection. The computer was equipped with an ATI Radeon HD 4670 graphics chip. The monitor had a resolution of $1920 \times 1080$ and a viewable diagonal measure of 21.5 inches.

\subsection{Color palette acquisition}

For each subject, we used a minimum motion task [Anstis and Cavanagh, 1983] to acquire a palette of colors motion-equiluminant with the background. 


\subsubsection{The minimum motion stimulus}

Four frames (comprising one full cycle) of the 8-frame stimulus used in this task are illustrated in Fig. 1. Each frame lasted 33ms. The outer and inner diameters of the motion annulus were 1.8 and 0.4 deg. respectively. Frame 1 is shown on the left of Fig. 1. In Frame 2, the square wave has been shifted one quarter cycle; if we think of this shift as clockwise (which corresponds to downward on the right side of Fig. 1), then the sections that were dark in Frame 1 have become green, and the sections that were light in Frame 1 have become gray. Frame 3 is identical to Frame 1, except that the dark and light bars switch roles, and Frame 4 is identical to Frame 2, except that the green and gray bars switch roles. Frames 5 through 8 repeat this pattern.

The method assumes that the motion of this stimulus is registered by only a single motion-sensing system in human vision. Although substantial evidence suggests that several different systems operate in human vision to detect motion [Lu and Sperling, 1995], the spatiotemporal properties of this stimulus $(7.58 \mathrm{~Hz}$ temporal frequency and $6 \%$ achromatic contrast) are chosen to activate exclusively the "first-order" system, i.e., the system that is sensitive to spatiotemporal modulations of luminance.

The logic of the minimum motion method is suggested by the light and dark arrows on the right side of Fig. 1. We assume that each of the four lights that occur in this stimulus (the dark-gray, the light-gray, the middle-gray and the green) produces a level of activation, that we will call "motion-luminance," in the unique system that registers the motion of this stimulus, and spatiotemporal variations in motion-luminance are analyzed for motion.

Suppose that the motion-luminance of the green light is higher than that of the middlegray light. In this case, models of first-order motion perception [Adelson and Bergen, 1985, van Santen and Sperling, 1984, 1985, Watson and Ahumada, 1985] predict that the motion evoked by the stimulus of Fig. 1 should follow the upward/counterclockwise path suggested by the white arrow in the right side of Fig. 1. Conversely, if the motion-luminance of the green light is lower than that of the middle-gray light, the motion evoked should follow the downward/clockwise path suggested by the black arrow.

By contrast, suppose the motion-luminance of the green light is equal to that of the middle-gray light. In this case, stimulus frames 2 and 4 become spatially uniform in motionluminance, in which case the stimulus devolves into pure flicker. The homogeneous motionluminance annuli that now occur in frames 2 and 4 serve merely to mark time between the patterns of motion-luminance occurring in frame 1 and 3 ; however, the pattern of motionluminance in frame 3 is merely the contrast-reversal of the pattern in frame 1, implying that this stimulus must evoke completely ambiguous motion.

\subsubsection{Estimating the equiluminant plane}

To estimate the equiluminant plane for a given subject, we constructed twenty sequences of lights, each of which followed a path around the saturation-perimeter of the set of lights achievable with our display system starting at a high-luminance light and ending at a lowluminance light. Different paths passed through different hue regions. For each of the 20 sequences, the subject first used the up and down arrows on the keyboard to move along the path to select the light $C_{\text {start }}$ that appeared (according to a minimum border contrast 


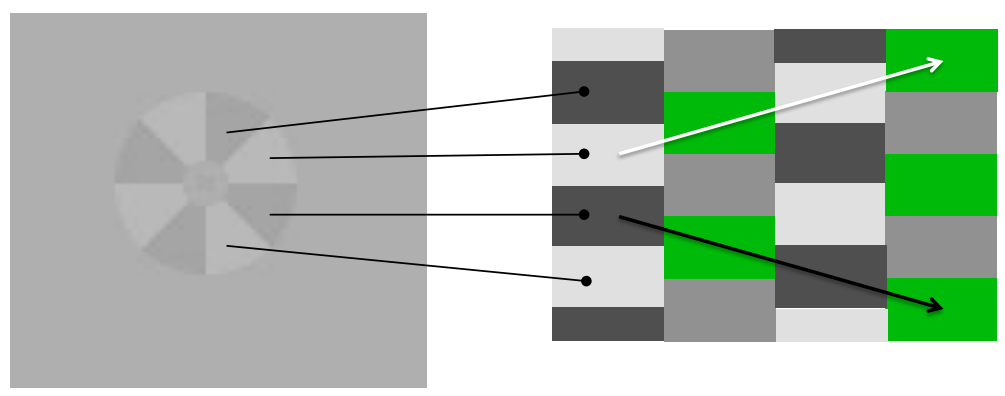

Figure 1: On the left, a static depiction of the moving stimulus. On the right, a vertical representation of the radial stimulus. Each column represents a frame (four vertical frames are depicted). Each frame is replaced by the adjacent, occupying the same location, so that a slight displacement of the gating is perceived as the frames are rapidly presented.

criterion) to be equiluminant with a standard gray light $G$ with luminance $52 \mathrm{~cd} / \mathrm{m}^{2}$.

Then the subject performed a series experimental trials in which he-or-she judged the direction of the motion evoked by the motion stimulus (Fig. 1). An up-down staircase was used to move along the path of lights starting at $C_{\text {start }}$. A cumulative normal psychometric function was fit to the resulting data to find the color along that path that was motionequiluminant to $G$.

We proceeded to find the plane in the space spanned by 3 color fundamentals of our monitor that best fit the 20 lights obtained from the subject. The direction normal to this plane was taken to be the luminance axis for the subject.

For the present experiment, we used a set of motion-equiluminant lights that isolated the constant- $S$ cardinal axis, cSCA, of DKL space. Specifically, the lights in this set

1. all produced equal activation in the (Stockman-Sharpe, 2 deg.) S-cone fundamental.

2. were all motion-equiluminant to the achromatic light $G$ with photometric luminance of $52 \mathrm{~cd} / \mathrm{m}^{2}$.

Eight lights were used for each subject. These lights projected onto locations $-4 \alpha,-3 \alpha$, $-2 \alpha,-\alpha, \alpha, 2 \alpha, 3 \alpha, 4 \alpha$, of the $L-M$ cardinal axis of DKL space, where $\alpha$ was chosen to be as large as possible on our display device given that location 0 corresponded to the achromatic light $G$ with photometric luminance of $52 \mathrm{~cd} / \mathrm{m}^{2}$. We will write $\Omega$ for the set containing these 8 colors.

The cone-contrasts (relative to $G$ ) of the lights used for different participants are shown in Fig. 2. In this figure, the lights used for a given participant follow a straight line which is the intersection of the equiluminant plane estimated for the participant and the plane of lights with S-cone contrast 0 . The participant number is at the right (green) end of the line.

It was a mistake on our part to leave a gap in our set of colors at $\alpha=0$. The lines plotted in Figs. 3a and 3b should be straight, but they are not straight because of this error. Similarly, in Figs. 4a and 4b should be parabolic, but they are too fat because of this error. However, this mistake does not compromise the logic of the study in any important way. 


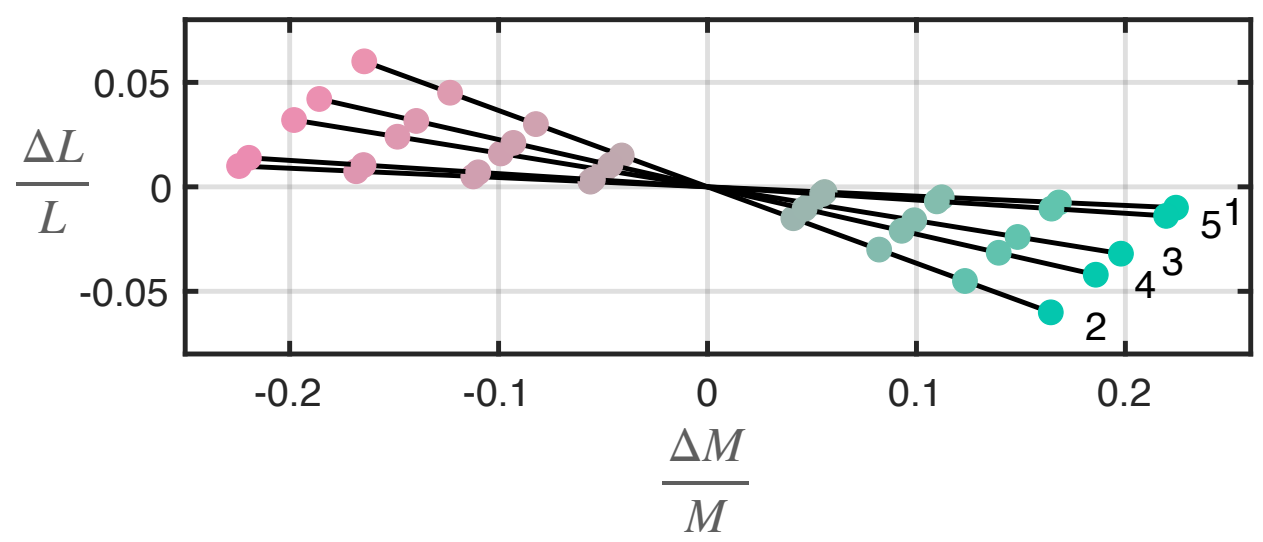

Figure 2: The cone contrasts of the lights used for the five participants. All cone contrasts are computed relative to the achromatic light with photometric luminance of $52 \mathrm{~cd} / \mathrm{m}^{2}$. For each participant, the line connecting the eight colors used for that participant is the intersection of the equiluminant plane estimated for that participant and the plane of lights with $S$-cone contrast 0 . The $L$ - and $M$-cone contrasts of the eight lights used for participant $n=1,2 \cdots, 5$ are marked by $n$ at the right end of the sequence of eight lights.

\section{$2.4 \Omega$-scrambles}

Stimuli were composed of chromatic textures called $\Omega$-scrambles. $\Omega$ is a set, in principle of arbitrary size, whose elements will be used to compose a texture; in practice, and for reasons that will become clear once we describe how the textures are constructed, the number should be small; in our case, the elements of $\Omega$ are a set of eight colors, chosen to be motionequiluminant via the method described in the previous section. An $\Omega$-scramble is a patch of texture comprising small square elements, each of which is colored with one of the colors in $\Omega$. The "histogram" of the scramble is the probability distribution that gives the proportions with which the different colors in $\Omega$ appear in the patch. In practice, to generate a patch of $\Omega$-scramble comprising $N$ spatial elements with histogram as close as possible to a given probability distribution $p$, we load a virtual urn with $N$ copies of colors drawn from $\Omega$ in proportions conforming as closely as possible to $p$. These colors are then assigned randomly without replacement to the $N$ locations of the patch.

We write $U$ for the uniform histogram (i.e., $U(\omega)=\frac{1}{n}$ for all $\omega \in \Omega$, where $n$ is the size of $\Omega$ ). In addition, we call any function $\rho: \Omega \rightarrow \mathbb{R}$ a perturbation if

1. $\sum_{\omega \in \Omega} \rho(\omega)=0$, and

2. $|\rho(\omega)| \leq \frac{1}{n}$ for all $\omega \in \Omega$.

These two conditions insure that $U+\rho$ and $U-\rho$ will both be probability distributions on $\Omega$. If in fact $|\rho(\omega)|=\frac{1}{n}$ for some $\omega \in \Omega$, we call $\rho$ a maximal perturbation.

We assume that human vision has some number $M$ of mechanisms that are differentially sensitive to $\Omega$-scrambles, and for $m=1,2, \cdots, M$, the sensitivity of mechanism $m$ can be characterized by a function $F_{m}(\omega)$ that reflects the activation produced in mechanism $m$ by a texture element of color $\omega$. We assume the space-average activation produced in mechanism 
$m$ by an $\Omega$-scramble with histogram $p$ is equal to the dot product of $f$ and $p$ :

$$
f \bullet p=\sum_{\omega \in \Omega} F_{m}(\omega) p(\omega)
$$

The difference in activation produced in mechanism $m$ by scrambles with histograms $p$ and $q$ is $F_{m} \bullet(p-q)$. When $p=U+\rho$ and $q=U-\rho$, the difference in activation is $2 F_{m} \bullet \rho$.

The goal in the current experiment is to determine the sensitivity functions of the mechanisms in human vision that are differentially sensitive to $\Omega$-scrambles.

\subsection{The basic search task}

Each stimulus comprised an annulus of $\Omega$-scramble with some histogram $U-\rho$ which contained a disk of $\Omega$-scramble with histogram $U+\rho$ at one of eight possible locations (corresponding to map directions N, NE, E, SE, S, SW W, NW). The subject's task was to indicate the target location. Examples of stimuli are shown in Figs. 3 and 4. Subjects viewed the display from an unrestrained distance of approximately $95 \mathrm{~cm}$. At this distance the outer diameter of the stimulus annulus subtended $6.8^{\circ}$ of visual angle, and each texture element subtended $0.11^{\circ}$ of visual angle.

Between trials, the monitor screen remained gray except for a small central cue spot, slightly brighter than the background, that remained visible throughout the experiment. The background gray $\left(52 \mathrm{~cd} / \mathrm{m}^{2}\right)$ was the same gray with respect to which the colors used for a given subject were motion-equiluminant. Each trial was initiated with a button press following which the screen remained gray for $500 \mathrm{~ms}$. The stimulus then appeared for 300 ms and was followed by the gray screen during which the subject used the number pad keys to indicate the location of the target disk. The mapping was: "7" for NW, "8" for N, "9" for NE, "6" for E, "3" for SE, "2" for S, "1" for SW, "4" for W. A beep sounded after an incorrect response.

\subsection{The four tasks}

Each subject performed 2600 trials in each of four, separately blocked tasks. These four tasks all required judgments of target location as described in Section 2.5. However, the predominant quality that differentiated the target from the background differed in different tasks. In task 1 the target was pinker than the background (Fig. 3c). In task 2 (Fig. 3d), the target and background colors were reversed. In task 3, the target was higher in red-green contrast than the background (Fig. 4c); in task 4, the target and background colors were reversed (Fig. 4d). Each of these tasks was an individual application of the "seed expansion" method [Chubb et al., 2012]. The next section gives a brief overview of the method as it was used in one of the four tasks in the current experiment.

\subsubsection{The use of the seed expansion method in the current experiment}

For task $t=1,2,3,4$, a single "seed" perturbation $\phi_{t}$ is used to produce the difference between the target disk vs the background annulus on each trial. On each trial in the task with seed $\phi_{t}$, a perturbation $\rho$ is generated that correlates strongly and positively 

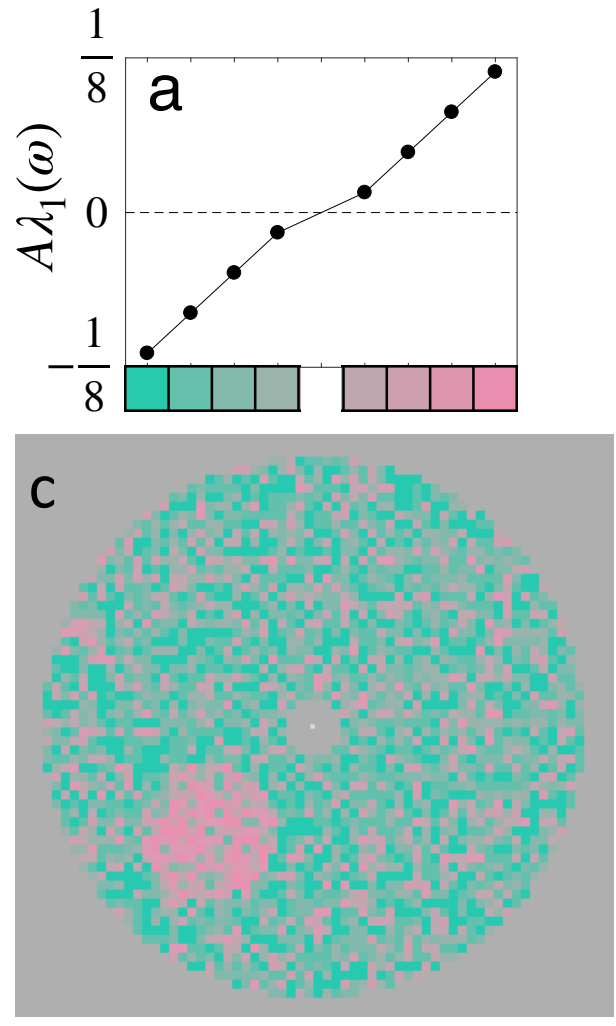
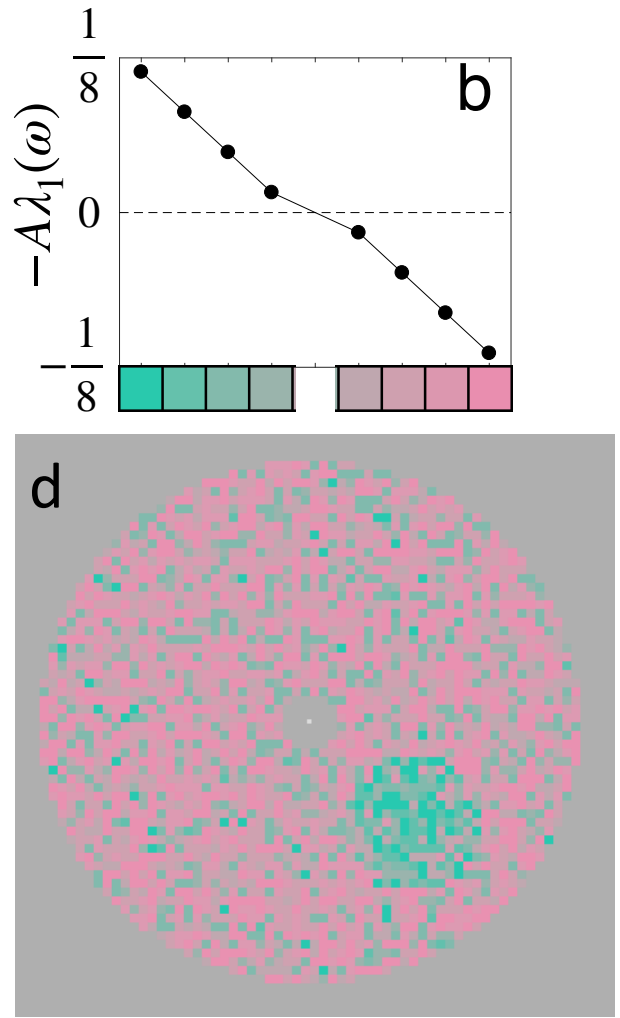

Figure 3: Examples of stimuli from complementary tasks 1 and 2 with perturbations (a) $\lambda_{1}$, and (b) $-\lambda_{1}$. (c) An example stimulus from task 1 ; the target disk is composed of $\Omega$ scramble with histogram $U+A \lambda_{1}$ and a background annulus with histogram $U-A \lambda_{1}$. The perturbation amplitude $A$ is chosen to make the perturbation $A \lambda_{1}$ maximal. (d) An example stimulus from task 2; The roles of the two different types of scramble have been reversed from (c). The targets in (c) and (d) are at locations Southwest and Southeast, respectively. 

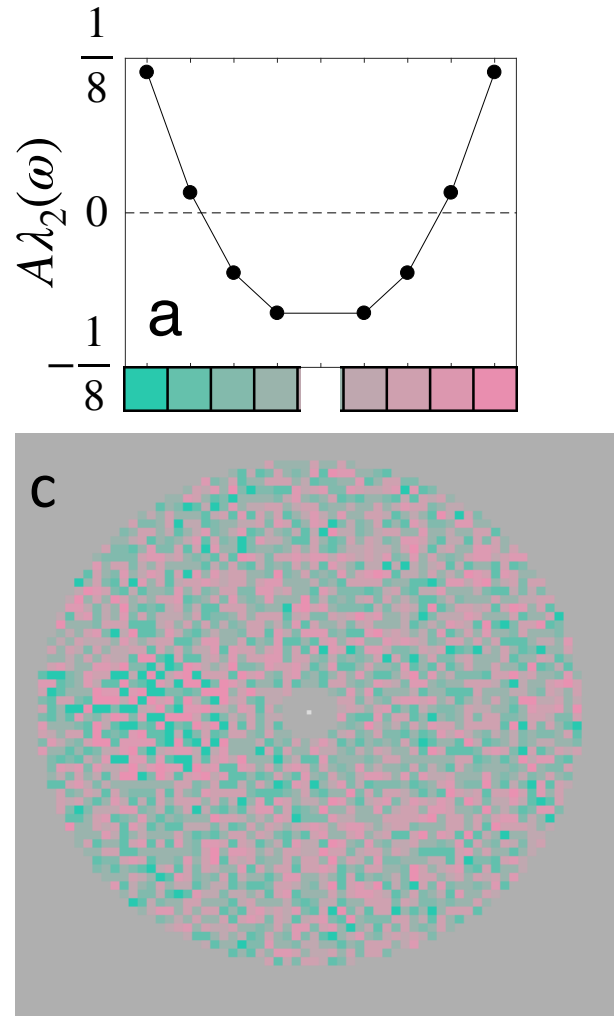
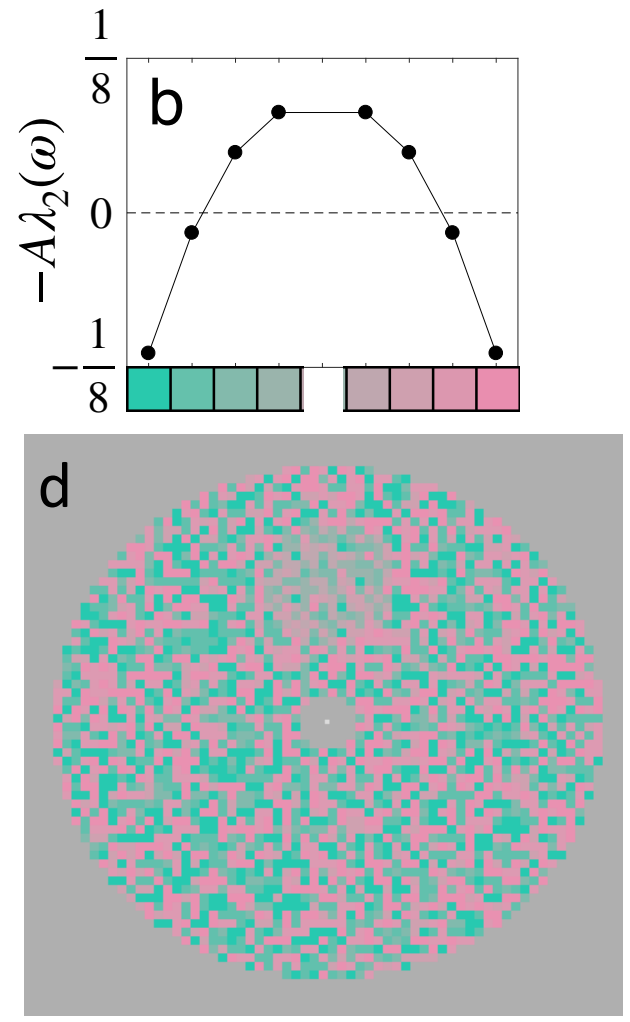

Figure 4: Examples of stimuli from complementary tasks 3 and 4 with perturbations (a) $\lambda_{2}$, and (b) $-\lambda_{2}$. (c) An example stimulus from task 3 ; the target disk is composed of $\Omega$ scramble with histogram $U+A \lambda_{2}$ and a background annulus with histogram $U-A \lambda_{2}$. The perturbation amplitude $A$ is chosen to make the perturbation $A \lambda_{2}$ maximal. (d) An example stimulus from task 4; The roles of the two different types of scramble have been reversed from (c). The targets in (c) and (d) are at locations West and North, respectively. 
with $\phi_{t}$. The $\Omega$-scramble that fills the target disk has a histogram $U+\rho$, and the annular background has the histogram $U-\rho$. Because each $\rho$ differs only slightly from the seed $\phi_{t}$, the qualitative difference between the target-disk and the background is similar from trial to trial. The point of separately blocking these different tasks is to encourage subjects to optimize their strategy for the particular target-vs-background texture difference in each task. In particular, our model assumes that in each task $t$, the subject $s$ combines information from his/her mechanisms to produce an task-specific tool $T_{s, t}$ that gives high values to colors $\omega \in \Omega$ that occur with high density in the target disk, and low values to colors $\omega \in \Omega$ that occur with low density in the background annulus in task $t$. We assume that the subject applies the tool $T_{s, t}$ to the stimulus on a given trial to produce a neural map in which the target location is highly activated in comparison to the background. In Chubb et al. [2012], the function $T_{s, t}$ is called the "expansion" of the seed perturbation $\phi_{t}$. Here we will call $T_{s, t}$ the "tool achieved by subject $s$ in task $t$ ".

Without making any assumptions about the nature of the mechanisms underlying performance, we can use a simple model to describe the tool $T_{s, t}$ achieved by subject $s$ in task $t$. This model assumes:

1. The salience of the target in task $t$ on a trial in which the target has histogram $U+\rho$ (and the background has histogram $U-\rho$ ) is

$$
\operatorname{Sal}_{s, t}(\rho)=T_{s, t} \bullet \rho .
$$

2. The probability of a correct response on such a trial is $\Psi_{s}(\operatorname{Sal}(\rho))$. $\Psi_{s}$ is a Weibull function defined by:

$$
\Psi_{s}(x)=0.125+0.855\left(1-\exp \left(-x^{B_{s}}\right)\right) .
$$

Note that for any given subject $s$, we assume that the steepness parameter $B_{s}$ is constant across different tasks. The estimated tools do not change appreciably if we allow the Weibull steepness parameters to vary across tasks for a given subject.

About $\Psi_{s}$, notice that

1. Chance performance is $\Psi_{s}(0)=0.125$ because the task requires an 8-option forced choice.

2. $\Psi_{s}$ asymptotes at 0.98 instead of 1.0 to cover the possibility of "finger errors," i.e., errors subjects make even though they clearly see correct response.

3. Typically, one expects a Weibull function to have two free parameters. However, there is only one $\left(B_{s}\right)$ in Eq. 5. This is because the other typical parameter (a scalar used to divide $x$ ) can be absorbed into the tool $T_{s, t}$ in Eq. 4 .

\subsubsection{The seed perturbations used in the four tasks}

To describe the perturbations used in these experiments, for $k=1,2, \cdots, 8$, let $\omega_{k}$ be the eight colors used in our scrambles ranging from green to red. For $j=0,1, \cdots, 7$, 
the discrete domain Legendre polynomials $\lambda_{j}\left(\omega_{k}\right)$ are derived by applying Gram-Schmidt orthonormalization to the sequence of monomials, $h_{j}\left(\omega_{k}\right)=k^{j}$. The Legendre polynomials of order $1,2, \cdots, 7$ are listed in table 2 in the Appendix.

The seed perturbations for tasks $1,2,3$ and 4 were $\phi_{1}=\lambda_{1}, \phi_{2}=-\lambda_{1}, \phi_{3}=\lambda_{2}$, $\phi_{4}=-\lambda_{2}$. (We had originally planned to also include tasks with seed perturbations $\lambda_{3}$, and $-\lambda_{3}$. However, these tasks proved too difficult.) Examples of stimuli from tasks 1 and 2 are shown in Fig. 3 and examples of stimuli from tasks 3 and 4 are shown in Fig. 4. (These example stimuli have the maximum possible histogram difference to make the texture differences characterizing the different tasks as obvious as possible.)

\subsubsection{Trial-by-trial perturbations within a given task}

With the exception of Subject 2 in task 1, each subject performed 2600 trials in each of the four tasks, 200 in each of 13 interleaved staircases. These 2600 trials were broken into 20 blocks, each of which included 10 trials from each of the 13 staircases. (In Task 1, Subject 2 completed only 19 blocks -190 trials in each of the 13 staircases.) This section describes the 13 staircases used in the different tasks. For a given task $t=1,2,3,4$, let $b_{1}=\phi_{t}$, and let

$$
b_{2}= \begin{cases}\lambda_{2} & \text { if } t \text { is } 1 \text { or } 2 \\ \lambda_{1} & \text { if } t \text { is } 3 \text { or } 4\end{cases}
$$

and for $k=3,4, \cdots, 7$, let $b_{k}=\lambda_{k}$. Then we construct the perturbations

$$
\eta_{k}^{+}=\frac{b_{1}+\frac{1}{2} b_{k}}{\left\|b_{1}+\frac{1}{2} b_{k}\right\|} \quad \text { and } \quad \eta_{k}^{-}=\frac{b_{1}-\frac{1}{2} b_{k}}{\left\|b_{1}-\frac{1}{2} b_{k}\right\|}
$$

for $k=2,3, \cdots, 7$. The $\eta_{k}+$ 's and $\eta_{k}^{-}$'s for tasks 1 and 3 are shown in Fig. 5 . Note that all of the perturbations $b_{1}$, and $\eta_{k}^{+}$and $\eta_{k}^{-}$for $k=2,3, \cdots, 8$ are normalized. Note also that the correlation between $\phi_{t}$ and each of $\eta_{k}^{+}$and $\eta_{k}^{-}$is 0.8944 .

For each of the 13 perturbations $\rho=b_{1}, \eta_{k}^{+}, \eta_{k}^{-}, k=2,3, \cdots, 7$, a staircase was used to test performance using perturbations $A \rho$ for various amplitudes $A$. Specifically, the staircase for a given perturbation $\rho$ could visit the 30 histogram amplitudes $A=\frac{A_{\max }}{30}, \frac{2 A_{\max }}{30}, \cdots, A_{\max }$, for $A_{\max }$ the scalar for which the maximum absolute value of $A_{\max } \rho$ is equal to $\frac{1}{8}$. Each staircase started at amplitude $A=A_{\max }$ and ran for 200 trials. In each staircase, $A$ was decremented whenever the previous two trials both yielded correct responses; otherwise $A$ was incremented. These 13 staircases were randomly interleaved to collect the 2600 trials of data in each of tasks $t=1,2,3,4$.

\section{Model Description and Results}

Access to the data and modeling code is provided at https://github.com/cfchubb/RedGreen-Axis-Mechanisms.

\subsection{The descriptive model}

We first use a simple descriptive model to reveal the structure in our data under weak assumptions. This model (captured by Eqs. 4 and 5 ) allows all of the different tools $T_{s, t}$ 


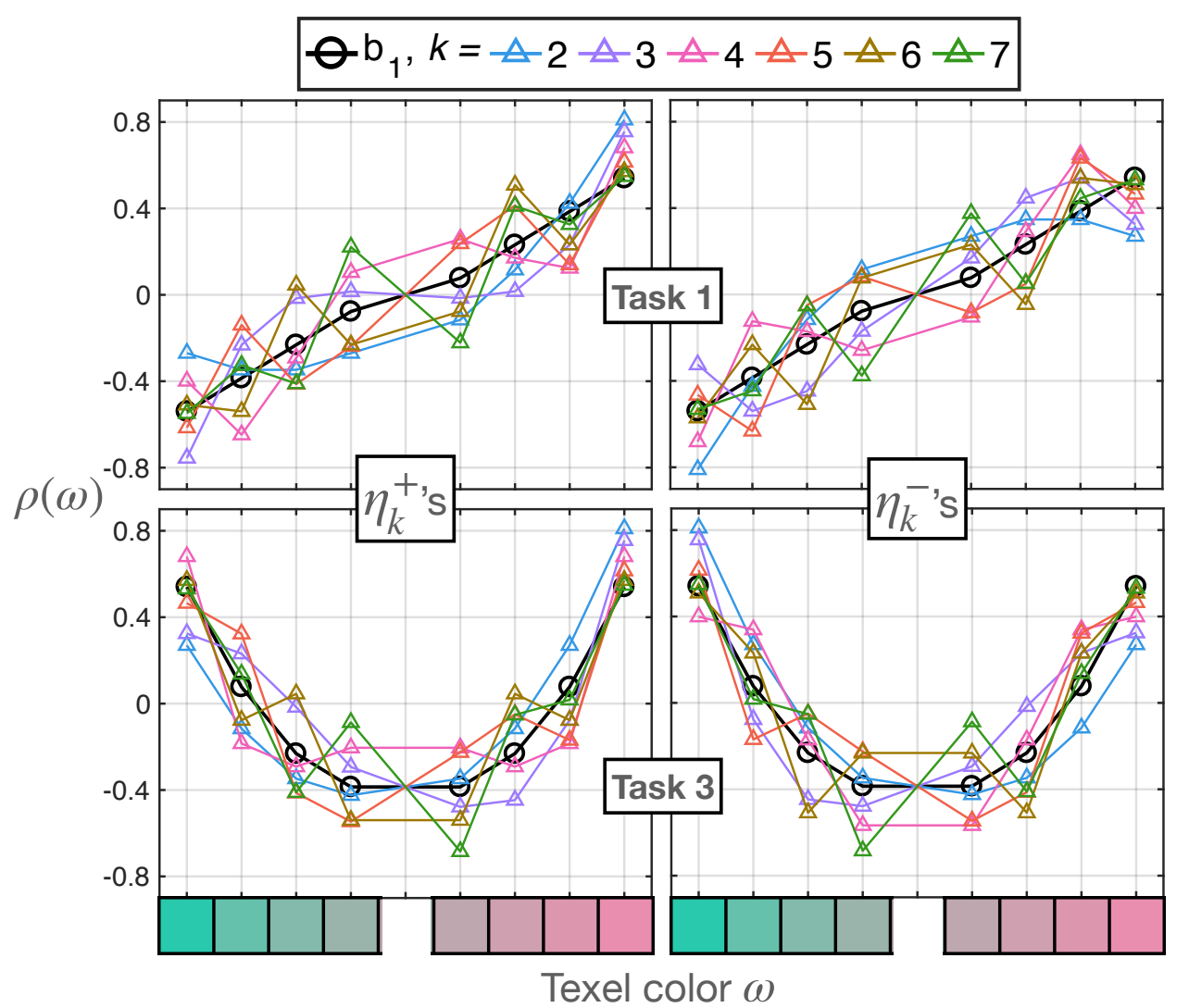

Figure 5: $\eta_{k}^{+}$'s and $\eta_{k}^{-}$'s for tasks 1 and 3. (Plots are analogous for task tasks 2 and 4.)

achieved by different subjects $s$ in different tasks $t$ to be freely determined under the single constraint that subject $s$ uses the same Weibull steepness parameter $B_{s}$ in all four tasks. (The fits do not differ perceptibly if we allow the Weibull parameters to vary freely across tasks for a given subject.) In addition to the parameter $B_{s}$, the other parameters for subject $s$ are the values $T_{s, t}(\omega)$ for all $\omega \in \Omega$ and $t=1,2,3,4$. However, any given tool $T_{s, t}$ is determined only up to an unmeasurable additive constant (which is equal to the mean activation produced in the tool by $\Omega$-scramble with histogram $U$ ). Thus, for a given subject this model has $4 \times 7+1=29$ free parameters; hence, including all five subjects, the model has $5 \times 29=145$ free parameters. The Bayesian procedure we use to fit the model is detailed in the Supplementary materials.

\subsection{Descriptive model results}

The results of the descriptive model for all subjects $s$ in all tasks $t$ are plotted by the white lines in Fig. 6. Notice that subjects do indeed achieve very different tools in the four different tasks. This demonstrates that subjects can flexibly deploy a range of different strategies in sensing differences between $\Omega$-scrambles.

We assume that in each different task $t$, subject $s$ has synthesized the tool $T_{s, t}$ out of the mechanisms available in his-or-her visual system specifically to optimize performance in task $t$. In order to gain insight into the nature of these underlying mechanisms, we will 
need to make specific assumptions about how subjects can combine the responses of different mechanisms to produce these tools. These assumptions are described in Section 3.3.

\subsection{The tool-construction model}

Our goal is to determine (1) the number $N_{m}$ of mechanisms (which will turn out to be 3 ) and (2) the sensitivity of each mechanism to the eight colors used in our stimuli; to derive the statistical power required to obtain clear answers to these questions, we will need to to pool the data from all five subjects across all four tasks. The following model enables this. We assume:

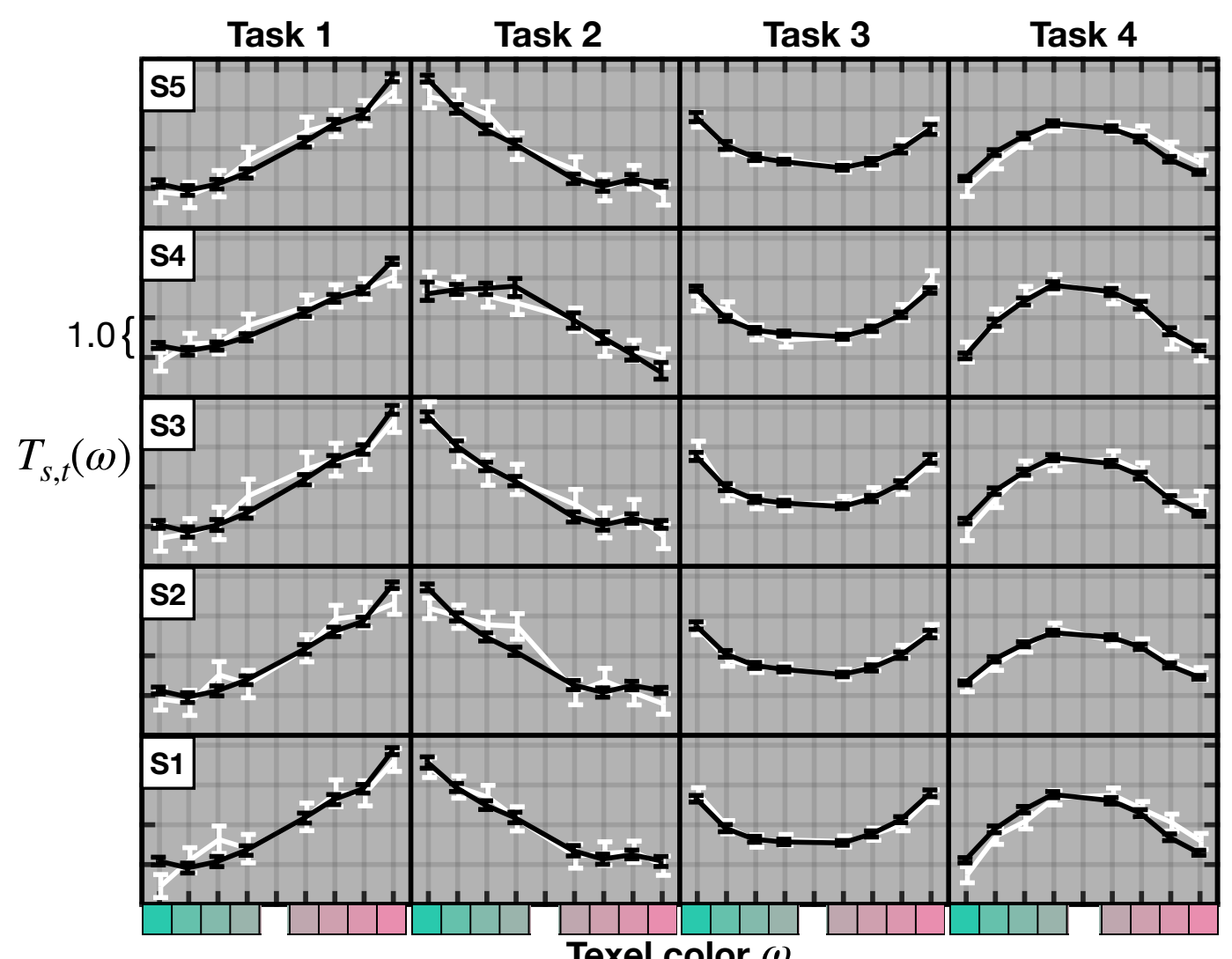

Figure 6: Tools achieved by all subjects in all tasks. Each row of panels corresponds to a subject $s$, and the tools $T_{s, t}$ achieved by that subject for tasks $t=1,2,3,4$ are plotted in successive columns. The white curve in each panel shows the form of $T_{s, t}$ yielded by the descriptive model. The black curve shows the form predicted by the tool-construction model assuming the three mechanisms shown in Fig. 7b. Each curve is defined only up to an arbitrary additive constant; accordingly, we limit the labeling of the tick marks to indicate only that the difference between successive tick marks is 1.0.

1. All subjects share the same set of mechanisms; however, the relative sensitivity of these mechanisms can differ between different subjects. Specifically, for $m=1,2, \cdots, N_{m}$, 
we assume that there exist functions $F_{m}: \Omega \rightarrow \mathbb{R}$, such that for any subject $s$, the sensitivity function $F_{s, m}(\omega)$ of mechanism $m$ for subject $s$ is given by

$$
F_{s, m}(\omega)=A_{s, m} F_{m}(\omega) \quad \text { for all } \omega \in \Omega
$$

for some scalar $A_{s, m}$. In order to uniquely determine the values $A_{s, m}$, we impose the constraint that $\left\|F_{m}\right\|=1$, for $m=1,2, \cdots, N_{m}$; under this constraint, we call $A_{s, m}$ the amplitude of mechanism $m$ for subject $s$.

2. The tool $T_{s, t}$ achieved by subject $s$ in the task $t$ is a weighted sum of his-or-her mechanism sensitivity functions:

$$
T_{s, t}(\omega)=\sum_{m=1}^{N_{n}} w_{s, t, m} F_{s, m}(\omega) \quad \text { for all } \omega \in \Omega
$$

3. The weights $w_{s, t, m}$ in Eq. 9 are chosen to maximize the performance of subject $s$ in task $t$ under the constraints that

$$
\sum_{m=1}^{N_{m}} w_{s, t, m}^{\beta_{s}}=1 \quad \text { and } \quad w_{s, t, m} \geq 0 \text { for } m=1,2, \cdots, N_{m},
$$

where the parameter $\beta_{s}$ controls the way in which subject $s$ is able to combine the responses of his-or-her different mechanisms.

\section{Comments on the assumptions:}

1. Because the weights $w_{s, t, m}$ are required to maximize performance of subject $s$ in task $t$ under the constraints imposed by Eq. 10, these weights are not free parameters; they are entirely determined by the other model parameters.

2. As suggested by Eq. 4, the performance of subject $s$ is optimized in task $t$ by choosing the weights $w_{s, t, m}$ to maximize

$$
T_{s, t} \bullet \phi_{t}
$$

3. The second constraint in Eq. 10 that the weights $w_{s, t, m}$ must be nonnegative reflects two assumptions:

- Mechanisms produce only nonnegative activations.

- Targets are localized by maxima in spatial distributions of neural activation.

These two assumptions jointly imply that only those mechanisms that give larger responses to the target texture than to the background texture are likely to be useful in any given task $t$. 
4. Concerning the combination parameter $\beta_{s}$ : We think of $\beta_{s}$ as constraining the possible strategies that are open to subject $s$. Suppose $\beta_{s}=1$; then the weights that $s$ can use to combine his-or-her mechanisms in any given task must sum to 1 . Under this constraint, in the task with target perturbation $\phi_{t}, s$ 's best option is always to put all his-or-her eggs in the single basket that gives the biggest payoff - i.e., to assign weight 1 to the single mechanism whose sensitivity function correlates most strongly with $\phi_{t}$. Giving any weight to any other mechanism lowers the response of the tool $T_{s, t}$ to $\phi_{t}$. At the other extreme, suppose $\beta_{s}$ is very large; then all of the weights $w_{s, t, m}$ can be made very close to 1 while keeping the sum of $w_{s, t, m}^{\beta_{s}}$ equal to 1 . In this case, it pays to boost the weights $w_{s, t, m}$ as much as possible to all the mechanisms whose sensitivity functions correlate positively with $\phi_{t}$ because all such boosts increase the response of $T_{s, t}$ to $\phi_{t}$. To summarize, if $\beta_{s} \leq 1$, then the subject's best strategy is always to select the single mechanism whose sensitivity function correlates most strongly with $\phi_{t}$. We therefore assume without loss of generality that $\beta_{s} \geq 1$. If $\beta_{s}=\infty$, then the subject's best strategy is always to combine all mechanisms whose sensitivity functions correlate positively with $\phi_{t}$ with equal weight. Intermediate values of $\beta_{s}$ dictate specific strategies between these extremes.

\subsection{Tool-construction model results}

\subsubsection{The 2-mechanism fails to account for the results from the descriptive model}

Under the assumption that human vision possesses only two mechanisms that are differentially sensitive to $\Omega$-scrambles, the tool-construction model fails to account for the data. The 2-mechanism fit is well-constrained by the data: multiple McMC processes with different starting locations all converge to the same posterior density, and the autocorrelation functions for these McMC processes drop to 0 for offsets greater than 4000; thus, independent samples from the posterior density are easily obtained. The normalized sensitivity functions (Fig. 7, panel (a)) are suggestive of red and green half-wave linear mechanisms. As shown by the black lines in Fig. 8, this model does a good job of predicting the tools achieved by our subjects in tasks 1,2 and 3. However, it fails to account for performance in task 4; as indicated by the flat black lines in Fig. 8, the 2-mechanism tool-construction model predicts chance performance in task 4 . However, subjects achieve tools in task 4 comparable in effectiveness to those they achieve in task 3. This makes it clear that at least three mechanisms are needed.

\subsubsection{The 4-mechanism tool-construction model is poorly constrained by the observed results}

The four-mechanism tool-construction model is poorly constrained by the data (Fig. 7c). Although two of the mechanism sensitivity functions (which correspond roughly to the mechanisms labeled "Green" and "LCC" in 7b) seem to be well-constrained by the data, the other two sensitivity functions (which roughly replace the mechanism labeled "Red" in Fig. 7b) are closely intertwined, ragged in form, and bracketed by large credible intervals, suggest- 

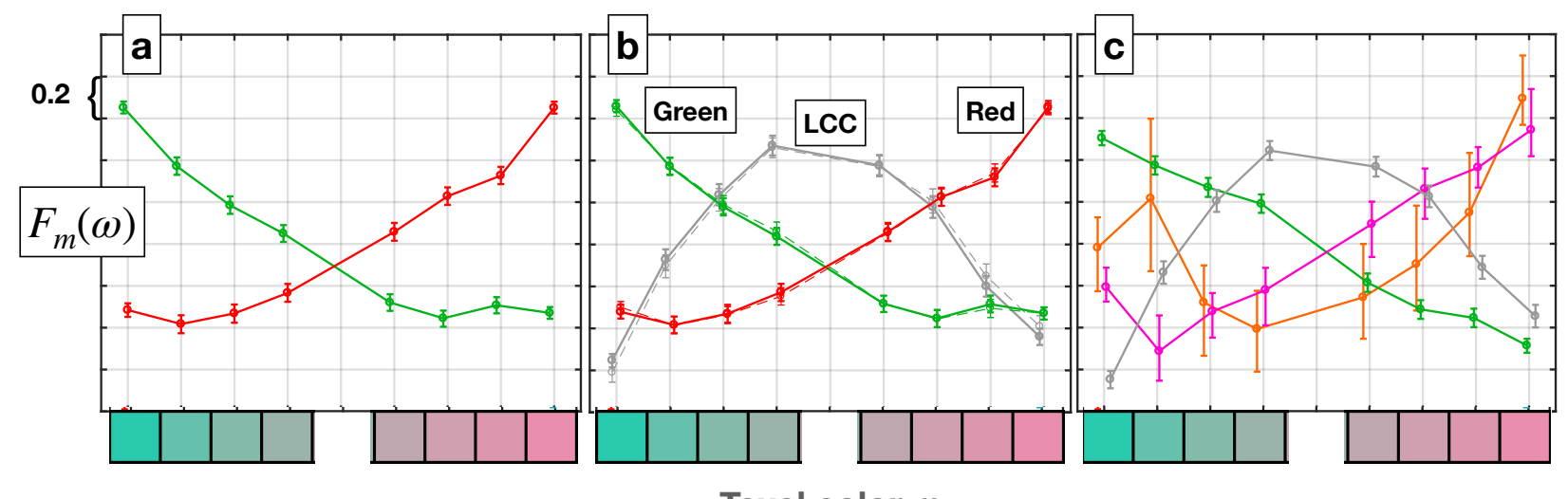

Texel color $\omega$

Figure 7: Mechanism functions $F_{m}$ under the assumption of (a) 2 mechanisms, (b) 3 mechanisms, (c) 4 mechanisms. The 2-mechanism fit cannot account for performance in task 4 (see Fig. 8). The 3-mechanism fit is bistable; the sensitivity functions predicted by the primary and secondary fits are plotted by the solid and dashed lines in panel (b). The 4-mechanism fit is poorly constrained by the data. Thus, three mechanisms are implicated: a green quasihalf-wave linear mechanism, a red quasi-half-wave linear mechanism and a "low chromatic contrast" (LCC) mechanism.

ing that these sensitivity functions are poorly constrained by the data. Despite extensive burn-in (over 6,000,000 iterations), including adjustment of Markov chain Monte Carlo process parameters for efficient sampling of the posterior distribution, trace plots for these two sensitivity functions show large-scale variability (Fig. 7 of the supplementary materials). Consequently, the autocovariance functions for the parameters in these functions (and the other model parameters associated with these functions) are well above zero over all lags.

\subsubsection{The 3-mechanism tool-construction model fit}

Interestingly, the 3-mechanism tool-construction model fit is bistable. A trace plot that shows this is shown in Fig. 4 of the Supplementary materials. This figure is derived from 64,000,000 McMC-iterations (post-burn-in, thinned to 640,000 by keeping every $100^{t} h$ sample). The individual traces in this plot correspond to the the 24 parameters included in the three normalized, mechanism sensitivity functions. As this figure reveals (perhaps most clearly in the bottom-most trace), the samples alternate between two distinct fits. The "primary" and "secondary" fits comprise roughly $80 \%$ and $20 \%$ of the samples, respectively. When we raise the number of mechanisms to 3 , the tool-construction model does a good job of capturing the tools achieved by all five subjects in all four tasks. The tools predicted by the 3 -mechanism model are plotted by the black lines in Fig. 6. The predicted tools account for $92 \%$ of the variance in the tools (estimated using the descriptive model) achieved by all subjects in all four tasks. The normalized mechanism sensitivity functions are plotted in panel (b) of Fig. 7. The "Green" mechanism is maximally activated by saturated green and shows decreasing activation with decreasing green saturation; this mechanism is invariant across the reds in $\Omega$. The "Low-Chromatic-Contrast" (LCC) mechanism shows maximal activation for gray and decreasing activation for increasingly saturated reds and greens. The "Red" mechanism is 




Figure 8: Tools predicted under the 2-mechanism model. Each column of panels corresponds to a subject $s$, and the tools $T_{s, t}$ achieved by that subject for tasks $t=1,2,3,4$ are plotted in successive rows. The white curve in each panel shows the form of $T_{s, t}$ yielded by the descriptive model. The black curve shows the form predicted by the tool-construction model assuming the two mechanisms shown in Fig. 7a. Each curve is defined only up to an arbitrary additive constant; accordingly, we limit the labeling of the tick marks to indicate only that the difference between successive tick marks is 1.0. Note that the two-mechanism model fits the data well for tasks 1,2 and 3 but fails for task 4 (for each subject, the predicted tool is flat implying chance performance in this task).

maximally activated by saturated red and shows decreasing activation with decreasing red saturation; this mechanism is largely invariant across the greens in $\Omega$.

We separated this trace (using a painstaking, by-eye procedure) into sequences populated separately by samples from the primary and secondary fits. For each of these sequences, the autocovariance function went to 0 for lags of 140 or greater (corresponding to lags of 14,000 
from the original McMC sequence). We therefore thinned each of the primary and secondary sample-sequences, retaining samples $1,141,281, \cdots$. Fig. 5 of the supplementary materials shows the trace plots for the thinned sample-sequences.

Fig. 7b plots the normalized mechanism sensitivity functions from the two fits. The solid (dashed) line shows the estimated functions for the primary (secondary) fit. In addition, Fig. 9 shows a caterpillar plot of the other parameters $\left(\beta_{s}, B_{s}\right.$ and $A_{s, m}$ for for all subjects $s$ and $m=$ Green, LCC and Red) from the primary and secondary, 3-mechanism, tool-construction model fits. As is clear, the primary and secondary fits are very similar.

\subsubsection{Secondary aspects of the 3-mechanism tool-construction model.}

We conclude that the best description of the current results is given by the three-mechanism, tool-construction model.

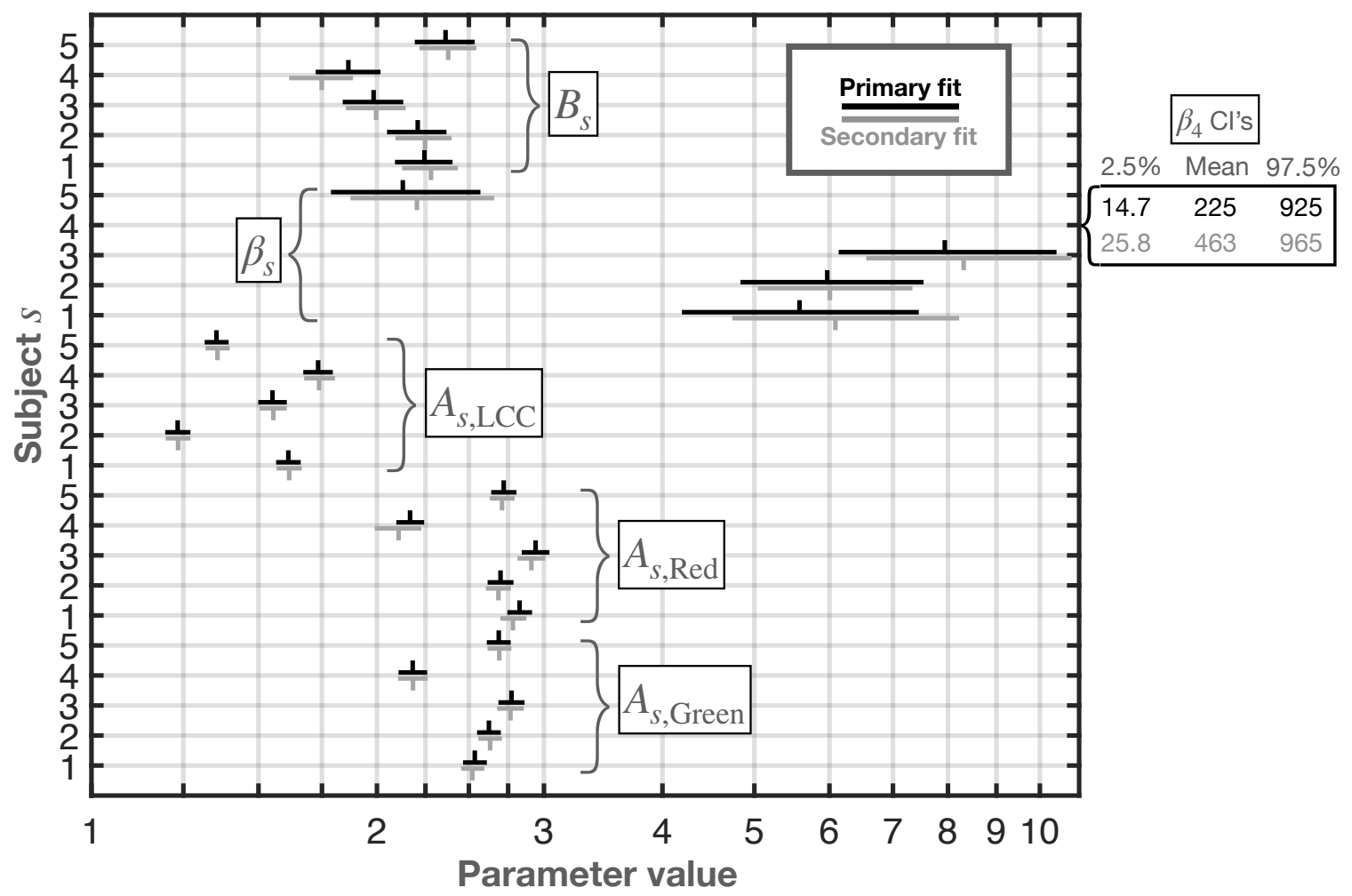

Figure 9: Caterpillar plot showing means (vertical bars) and 95\% credible intervals (horizontal bars) for parameters from the 3-mechanism tool-construction model. Not shown are the the normalized mechanism sensitivity functions, $F_{\text {Green }}, F_{\mathrm{LCC}}$, and $F_{\mathrm{Red}}$, which are shown in Fig. 7b. Black (gray) lines show "primary" ("secondary") fits. Note that the values for $\beta_{4}$ are off the scale and indicated in the box on the right.

There are several things to note about Fig. 9. First, the amplitude of the LCC mechanism tends to be lower than the amplitudes of the Green and Red mechanisms. Across all subjects, the mean LCC-mechanism sensitivity function amplitude is 1.49 as compared to 2.56 for the Green and 2.65 for Red. 


\begin{tabular}{|c|c|c|c|}
\hline subject & $A_{s, \text { Green }}$ & $A_{s, \mathrm{LCC}}$ & $A_{s, \text { Red }}$ \\
\hline 1 & $2.53 \pm 0.29$ & $1.61 \pm 0.27$ & $2.81 \pm 0.34$ \\
\hline 2 & $2.62 \pm 0.31$ & $1.23 \pm 0.21$ & $2.69 \pm 0.33$ \\
\hline 3 & $2.77 \pm 0.33$ & $1.55 \pm 0.26$ & $2.93 \pm 0.36$ \\
\hline 4 & $2.18 \pm 0.26$ & $1.73 \pm 0.29$ & $2.15 \pm 0.28$ \\
\hline 5 & $2.68 \pm 0.31$ & $1.35 \pm 0.23$ & $2.71 \pm 0.33$ \\
\hline
\end{tabular}

Table 1: The amplitudes of each of the Green, LCC and Red mechanisms in all five subjects. Note that the Green and Red mechanisms have roughly twice the amplitude of the LCC mechanism for all subjects.

We note in passing that the mechanism-combination parameter, $\beta_{s}$, is near 2 for subject $s=5$ but is greater than 5 for subjects $s=1,2,3$ and 4 . Thus, for subject 2, mechanisms combine in a near-Euclidean fashion. For the other subjects, however, (and especially for subject 4) the optimal tool in any given task was derived by giving similar weight to all functions $F_{s, m}$ that correlated positively with the target perturbation $\phi_{t}$.

Finally, we note that the curve for each of $F_{\text {Green }}$ and $F_{\text {Red }}$ appears to give higher weight to achromatic gray than it does to reddish (greenish) colors. However, because we omitted achromatic gray from $\Omega$, we have no actual estimate of the sensitivity of either of these mechanisms to this color.

\section{Discussion}

\subsection{Overview: Half-axis systems}

The visual system, after the rod and cone receptors, represents positive and negative quantities in different neurons (e.g., Kuffler's white-spot sensitive ON cells and dark-spot sensitive OFF cells) because that is enormously more energy-efficient than modulating a steady output up and down. In the DKL color space, the red-green axis is represented by a half-axis red-sensitive mechanism and a half-axis green-sensitive mechanism. Consider stimuli that contain contain equal amounts of red and green light, such as those in Fig. 4. When viewed from a distance at which the differently colored areas are unresolvable, both of these stimuli look uniformly gray. In the DKL system, subtraction of Green half-axis minus Red half-axis outputs represents hue, which in the case of equal outputs is neutral yellow or-if blue is present-white. Addition of the Green half-axis and red half-axis outputs represents intensity (luminance). In a yellow stimulus, subjects do not have separate access to the green and red half-axes. However, when the stimuli of Fig. 4 are viewed normally, subjects can estimate both the amount of red and the amount of green. Reporting the separate output of each half-axis system is a more complex computation that involves the histograms of the distribution of reds and greens, versus simply the net quantity of photons.

An interesting property of the derived mechanisms, is that they naturally form two opposing half-axis systems. The green-sensitive and red-sensitive mechanisms are analogous to the mechanisms in DKL space. On the other hand, the green+red tool and the LCC mechanism represent the two half-axes of a high-variance versus low variance continuum. 


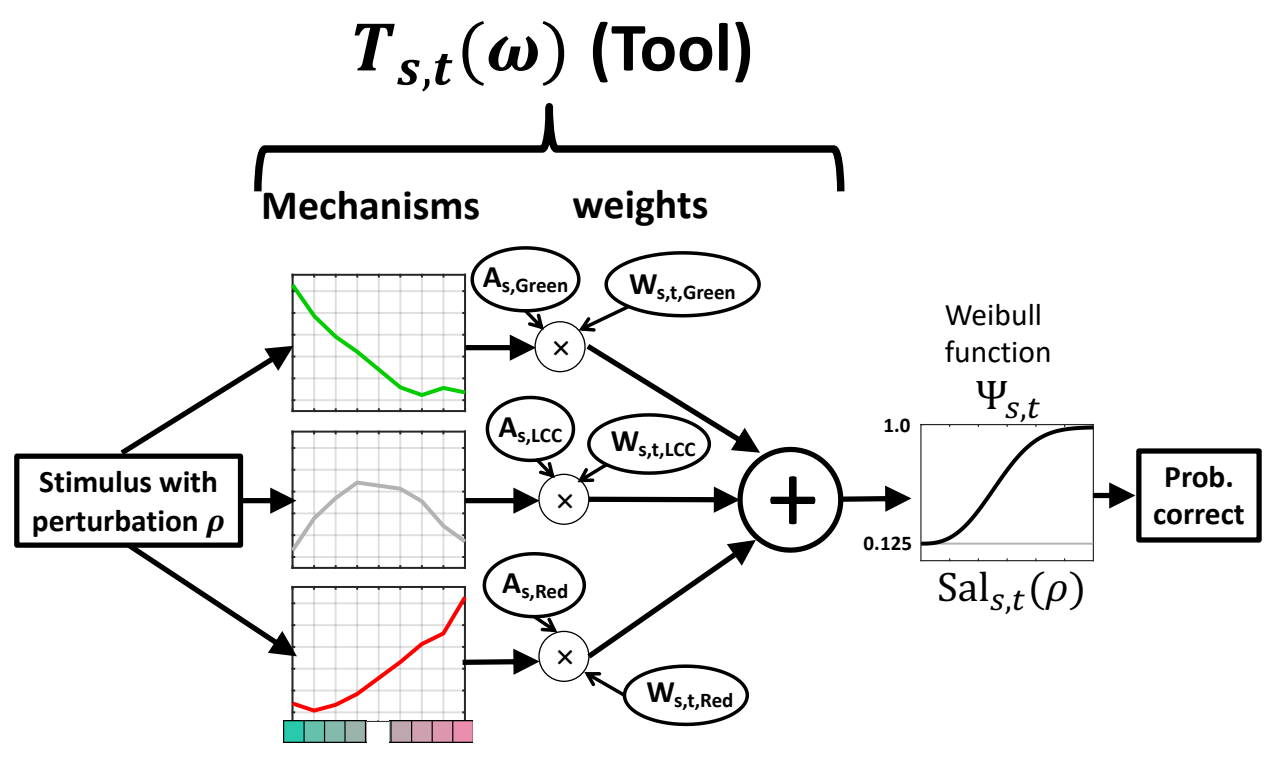

Figure 10: Schematic diagram of the 3-mechanism tool-construction model. The model predicts probability of a correct response by a given subject $s$ to a stimulus with perturbation $\rho$ in task $t$. The green, gray and red curves are the normalized mechanism sensitivity functions $F_{\text {Green }}, F_{\mathrm{LCC}}$ and $F_{\text {Red }}$. For a given one of these three mechanisms $M$, the mechanism sensitivity function for subject $s$ is then $A_{s, M} F_{M}$ (Eq. 8). For $\phi_{t}$ the target perturbation used in task $t$, the model assumes that the weights $w_{s, t, \text { Green }}, w_{s, t, \mathrm{LCC}}$ and $w_{s, t, \text { Red }}$ are chosen by subject $s$ to optimize performance in task $t$ by maximizing $\operatorname{Sal}_{s, t}\left(\phi_{t}\right)=T_{s, t} \bullet \phi_{t}$ under the constraints given in Eq. 10. A Weibull function converts $\operatorname{Sal}_{s, t}(\rho)$ to the predicted probability of a correct response.

Although not considered explicitly here, the mechanism for separating potential targets from background requires comparing parameters of the target and background saturation distributions. To solve general problems like those in Fig. 4 that involve distributions with equal luminance but different saturation distributions, both half-axis high-variance and lowvariance mechanisms are necessary. The end result of all the complications in the manuscript is the simplest (and quite plausible) set of mechanisms that can accomplish these detection tasks.

\subsection{Are the results consistent with previous findings?}

The current results suggest that three mechanisms in human vision are differentially sensitive to scrambles composed of lights drawn from the constant- $S$ axis of DKL space. A Red mechanism is activated in a graded fashion by lights of increasing red saturation; a complementary Green mechanism is activated in a graded fashion by lights of increasing green saturation. The third (LCC) mechanism enables detection of low-chromatic-contrast scrambles in highchromatic-contrast backgrounds. (An outline of the 3-mechanism, tool-construction model is shown in Fig. 10.)

This conclusion might seem to be at odds with results of Shepard et al. [2016] and Shepard 
et al. [2017]. In order to account for the pattern of chromatic detection thresholds in the presence of chromatic masking noise (modulated in different directions in color space), these authors posit six chromatic mechanisms. At least two of these mechanisms (the "red" and "orange" mechanisms) should be differentially activated by the reddish lights used in the current study. Similarly, at least two other of these mechanisms (the "green" and "blue" mechanisms) should be differentially activated by the greenish lights used in the current study. This raises the question: Do the current results contradict the theory of Shepard et al. [2016] and Shepard et al. [2017]?

The answer is, not necessarily. For concreteness we focus on the red and orange mechanisms; analogous comments apply to the green and blue mechanisms. Suppose the red and orange mechanisms share the same normalized sensitivity function $F(\omega)$ to the lights $\omega \in \Omega$ but that for any given subject $s$, the mechanism sensitivity functions $F_{s, \text { red }}(\omega)=A_{s, \text { red }} F(\omega)$

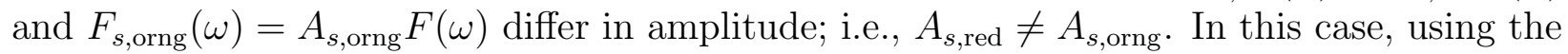
results of Sec. 4.4, it can be shown that a Tool-construction model that includes both the red and orange mechanisms can be replaced by an equivalent Tool-construction model that includes only one mechanism with normalized sensitivity function $F$. Under this model, the sensitivity function of this mechanism for a given subject $s$ is $F_{s, \text { both }}(\omega)=A_{s \text {,both }} F(\omega)$ for

$$
A_{s, \text { both }}=\left(A_{s, \text { red }}^{\frac{1}{\beta_{s}-1}}+A_{s, \text { orng }}^{\frac{1}{\beta_{s}-1}}\right)^{\beta_{s}-1} .
$$

This implies that the Tool-construction model can only discover mechanisms whose normalized sensitivity functions are not scalar multiples of each other. Thus, if the functions characterizing the sensitivity of the red and orange mechanisms to the lights $\omega \in \Omega$ are too similar in form, the current experiment will be unable to differentiate them. However, if the red and orange mechanisms differ strongly enough in the patterns of their sensitivity to the lights in $\Omega$, then the current experiment should have revealed this. We conclude that the red and orange mechanisms share a similar pattern of sensitivity to the lights in $\Omega$.

A related question is the following: What is the upper limit on the number of mechanisms that we could possibly have discovered in the current experiment. The answer is 7 . This is the number of linearly independent functions, all with mean 0 , that can be defined on our set $\Omega$, which includes 8 different lights.

\subsection{The nature of the LCC mechanism}

In accord with previous research [Krauskopf et al., 1982, Gegenfurtner and Kiper, 1992, Giulianini and Eskew, 1998, Hansen and Gegenfurtner, 2006, 2013, Shepard et al., 2016, Sankeralli and Mullen, 2001], we interpret the red and green half-wave linear mechanisms as sensitive to variations in the average spectral properties of the light impinging on the retina. It is tempting to interpret the curve for the LCC mechanism similarly. Under this interpretation, the LCC mechanism would be maximally activated by achromatic light and would show decreasing activation for increasingly saturated red and green lights. To our knowledge, previous research gives no support to such an interpretation. 


\subsubsection{Is the LCC mechanism a luminance artifact?}

We wondered whether the LCC mechanism might be due to a difference in luminance between the low vs. high chromatic contrast textures used in Task 4. The following considerations suggested that this might be the case: the minimum-motion stimulus used to insure that the lights used in our stimuli were equiluminant was a fairly small, centrally presented annulus (outer diameter $1.8^{\circ}$, inner diameter $0.4^{\circ}$ ), but our test stimuli were all presented extrafoveally. In particular, our target texture patches were presented between $2^{\circ}$ and $5.4^{\circ}$ within a background annulus that ranged from $0.68^{\circ}$ and $6.8^{\circ}$. At eccentricities beyond around $5^{\circ}$ or $6^{\circ}$, the Stockman-Sharpe $L$ - and $M$-cone fundamentals cease to be valid (because of lower density macular pigment and lower optical density cone photopigments due to shortened outer segments). However, luminance depends on the $L$ - and $M$-cones. This raises the possibility that, due to their extrafoveal presentation, the low-chromatic-contrast target disks used in Task 4 might differ in luminance from the high-chromatic-contrast backgrounds in which they occurred. To investigate this question, we tested a single subject with the minimum motion stimulus described in Sec. 2.3.1 centered (1) in the fovea and (2) $3.7^{\circ}$ to the right of fixation (the eccentricity of the center of the target texture patch in our scramble stimuli). In both configurations, we obtained maximally saturated reddish and greenish lights (roughly aligned with the constant- $S$ axis) that were equiluminant with the background gray used throughout the experiments. When rounded to the nearest pixelvalue, the reddish lights estimated to be equiluminant to the background gray light using the centrally and peripherally presented motion stimulus were identical. The same was true for the greenish lights. We conclude that the LCC mechanism is not due to a difference in luminance between our high vs. low chromatic contrast textures.

\subsubsection{The LCC mechanism may be a "second-order" chromatic mechanism.}

We suspect that the LCC mechanism is a "second-order" chromatic mechanism, sensitive not to the space-average spectral properties of light but rather to the local spatial pattern of the light on the retina. In particular, as suggested by the name "low chromatic contrast mechanism," we hypothesize that this mechanism is activated by stimulus regions that are homogeneous in color and suppressed by regions of high chromatic variation.

\subsection{The role of top-down attention in controlling performance across tasks}

The tool-construction model builds on previous research suggesting that subjects can use top-down attention to achieve response statistics selectively sensitized to different colors as task demands are varied [D'Zmura, 1991, D'Zmura et al., 1997, D'Zmura and Knoblauch, 1998, Drew et al., 2010, Sun et al., 2016]. However, the tool-construction model goes a step beyond previous models in assuming that when faced with a particular task, participants can linearly combine their available mechanisms to synthesize a "tool" that is optimal for that task. This tool itself operates like a mechanism in the sense that it is differentially sensitive to different color textures that must be discriminated in the task for which it has been created, and the salience of the target in a given stimulus depends on the correlation 
of the histogram of that target with the sensitivity function characterizing the tool.

This model is very different from that of Shepard et al. [2016], for example, which is used to predict performance in tasks in which the participant strives to judge which of two intervals of chromatic noise (modulated in a particular direction in color space) contains a Gaussian blob of a given target color. All conditions in their experiment are separately blocked. This feature of the design might well be expected to encourage participants to make use of topdown attention (if possible) to optimize performance in individual conditions. However, the model of Shepard et al. [2016] assigns no role to top-down attention. In particular, the probability of a correct response in a given condition depends only on the sensitivities of the different mechanisms to the noise variations and to the signal color in that condition. The salience of the target in the noise is assumed to result from a Minkowski combination (with an exponent of 4) of the individual mechanism responses.

The contrast between the tool-construction model and the model of Shepard et al. [2016] raises a fundamental question: To perform tasks of the sort used by Shepard et al. [2016], do participants synthesize different tools for different tasks, or do they rely on a fixed computation that always combines mechanism responses in the same way? There is an important difference between the predictions made by the tool-construction model versus the model of Shepard et al. [2016]. Consider a task of the sort used by Shepard et al. [2016] with a mean target light direction $D$ away from the white point in color space to be detected in some chromatic masking noise $N$. Imagine, however, that on different trials, the target light direction is perturbed slightly away from $D$; thus, on different trials the direction is $D+\theta$ for angular perturbations $\theta$ that are kept small enough to encourage the participant to use an invariant decision statistic across trials. Imagine further that adaptive procedures are used to concentrate trials across a set of lights $L$ in in this pie-slice of directions that support near-threshold performance.

The tool-construction model predicts that in such a task, the influence exerted on the participant's judgements by should vary linearly across the lights $L$. This predicted linearity follows from the fact that the tool synthesized by the participant is a linear combination of mechanism sensitivity functions and therefore equivalent to a "task-specific mechanism." The gradient of this linear function reflects the direction in color-space of the tool, and the equiperformance contours are linear/planar and normal to this gradient. By contrast, for tasks in which at least two mechanisms are predicted to contribute significantly to performance, the model of Shepard et al. [2016] predicts that the influence exerted on the participant's judgements should vary nonlinearly across the lights $L$. In particular, the equiperformance contours should follow a convex curve reflecting the change in predominance (with color direction) of the different mechanisms contributing to performance.

\section{References}

E. H. Adelson and J. R. Bergen. Spatio-temporal enegry models for the perception of apparent motion. Journal of the Optical Society of America A, 2:284-299, 1985.

S. Anstis and P. Cavanagh. A minimum motion technique for judging equiluminance. In 
J. D. Mollon and E. T. Sharpe, editors, Colour Vision, pages 155-166. Academic Press, 1983.

R. A. Bone and J. T. Landrum. Heterochromatic flicker photometry. Archives of Biochemistry and Biophysics, 430(2):137-142, 2004.

C. Chubb, I. Scofield, C-C. Chiao, and G. Sperling. A method for analyzing the dimensions of preattentive visual sensitivity. Journal of Mathematical Psychology, 56:427-443, 2012.

A. M. Derrington, J. Krauskopf, and P. Lennie. Chromatic mechanisms in lateral geniculate nucleus of macaque. Journal of Physiology, 357:241-265, 1984.

S. Drew, C. Chubb, and G. Sperling. Precise attention filters for weber contrast derived from centroid estimations. Journal of Vision, 10(10:20):1-16, http://www.journalofvision.org/content/10/10/20, 2010.

M. D’Zmura. Color in visual search. Vision Research, 31(6):951-966, 1991.

M. D’Zmura and K. Knoblauch. Color in visual search. Vision Research, 38:3117-3128, 1998.

Michael D'Zmura, Philippe Colantoni, Kenneth Knoblauch, and Bernard Laget. Color transparency. Perception, 26(4):471-492, 1997.

A. Eisner and D. I. MacLeod. Blue sensitive cones do not contribute to luminance. Journal of the Optical Society of America, 70:121-123, 1980.

R. T. Eskew. Higher order color mechanisms: A critical review. Vision Research, 49:26862704, 2009.

R. T. Eskew, J. R. Newton, and F. Giulianini. Chromatic detection and discrimination analyzed by a bayesian classifier. Vision Research, 41:893-909, 2001.

K. R. Gegenfurtner and D. C. Kiper. Contrast detection in luminance and chromatic noise. Journal of the Optical Society of America A, 9:1880-1888, 1992.

F. Giulianini and R. T. Eskew. Chromatic masking in the (dl:l, dm:m) plane of cone-contrast space reveals only two detection mechanisms. Vision Research, 38:3913-3926, 1998.

P. D. Gowdy, C. F. Stromeyer, and R. E. Kronauer. Detection of flickering edges: absence of a red-green edge detector. Vision Research, 39(25):4186-4191, 1999a.

P. D. Gowdy, C. F. Stromeyer, and R. E. Kronauer. Facilitation between the luminance and red-green detection mechanisms: enhancing contrast differences across edges. Vision Research, 39(24):4098-4112, 1999 b.

N. Graham. Visual Pattern Analyzers. Oxford University Press, New York, 1989.

S. L. Guth, J. V. Alexander, J. I. Chumbly, C. B. Gillman, and M. M. Patterson. Factors affecting luminance additivity at threshold among normal and color-blind subjects and elaborations of a trichromatic-opponent color theory. Vision Research, 8:913-928, 1968. 
T. Hansen and K. R. Gegenfurtner. Higher level chromatic mechanisms for image segmentation. Journal of Vision, 6(3:5):239-259, http://www.journalofvision.org/content/6/3/5, doi:10.1167/6.3.5., 2006 .

T. Hansen and K. R. Gegenfurtner. Higher order color mechanisms: Evidence from noise-masking experiments in cone contrast space. Journal of Vision, 13(1:26):1-21, http://www.journalofvision.org/content/13/1/26, doi:10.1167/13/1/26., 2013.

H. E. Ives. Studies of the photometry of lights of different colours. i. spectral luminosity curves obtained by the equality of brightness photometer and the flicker photometer under similar conditions. Philosophical Magazine, 24:149-188, 1912a.

H. E. Ives. Studies of the photometry of lights of different colours. ii. spectral luminosity curves by the method of critical frequency. Philosophical Magazine, 24:352-370, 1912b.

H. E. Ives. Studies of the photometry of lights of different colours. iii. distortions in spectral luminosity curves produced by variations in the character of the comparison standard and of the surroundings of the photometric field. Philosophical Magazine, 24:744-751, 1912c.

H. E. Ives. Studies of the photometry of light of different colours. iv. the addition of luminosities of different color. Philosophical Magazine, 24:845-853, 1912d.

H. E. Ives. Studies of the photometry of light of different colours. v. the spectral luminosity of the average eye. Philosophical Magazine, 24:853-863, 1912e.

H. E. Ives. The theory of the flicker photometer. Philosophical Magazine, 28:708-728, 1914.

H. E. Ives. A polarization flicker photometer and some data of theoretical bearing obtained with it. Philosophical Magazine, 33:360-380, 1917.

P. K. Kaiser. Minimally distinct border as a preferred psychophysical criterion in visual heterochromatic photometry. Journal of the Optical Society of America, 61(7):966-971, 1971.

P. K. Kaiser, B. B. Lee, P. R. Martin, and A. Valberg. The physiological basis of the minimally distinct border demonstrated in the ganglion cells of the macaque retina. Journal of Physiology, 422:153-183, doi: 10.1113/jphysiol.1990.sp017978, 1990.

J. Krauskopf and Q. Zaidi. Induced desensitization. Vision Research, 26(5):759-762, 1986.

J. Krauskopf, D. R. Williams, and D. W. Heeley. Cardinal directions of color space. Vision Research, 26:23-32, 1982.

J. Krauskopf, D. R. Williams, D. W. Heeley, and A. M. Brown. Higher order color mechanisms. Vision Research, 36:1235-1245, 1986.

H. De Lange. Research into the dynamic nature of the human fovea-cortex systems with intermittent and modulated light ii phase shift in brightness and delay in color perception. Journal of the Optical Society of America, 48:784-789, 1958. 
A. Li and P. Lennie. Mechanisms underlying segmentation of colored textures. Vision Research, 37:83-97, 1997.

Z.-L. Lu and G. Sperling. The functional architecture of human visual motion perception. Vision Research, 35:2697-2722, 1995.

D. I. A. MacLeod and R. M. Boynton. Chromaticity diagram showing cone excitation by stimuli of equal luminance. Journal of the Optical Society of America, 69(8):1183-1185, 1978.

K. T. Mullen and J. J. Kulikowski. Wavelength discrimination at detection threshold. Journal of the Optical Society of America A, 7:733-742, 1990.

J. R. Newton and R. T. Eskew. Chromatic detection and discrimination in the periphery: A postreceptoral loss of color sensitivity. Visual Neuroscience, 20:511-521, 2003.

C. Ripamonti, W. L. Woo, E. Crowther, and A. Stockman. The s-cone contribution to luminance depends on the $\mathrm{m}$ - and l-cone adaptation levels: Silent surrounds? Journal of Vision, 9(3:10):1-16, 2009.

W. A. H. Rushton. Pigments and signals in color vision. Journal of Physiology, 220(3): $1-31 \mathrm{P}, 1972$.

M. J. Sankeralli and K. T. Mullen. Postreceptoral chromatic detection mechanisms revealed by noise masking in three-dimensional cone contrast space. Journal of the Optical Society of America A, 14:2633-2646, doi:10.1364/JOSAA.14.002633, 1997.

M. J. Sankeralli and K. T. Mullen. Bipolar or rectified chromatic detection mechanisms? Visual Neuroscience, 18(1):127-135, 2001.

T. G. Shepard, E. A. Swanson, C. L. McCarthy, and R. T. Eskew. A model of selective masking in chromatic detection. Journal of Vision, 16(9:3):1-17, doi:10.1167/16.9.3., 2016.

T. G. Shepard, S. I. Lahlaf, and R. T. Eskew. Labeling the lines: A test of a six-mechanism model of chromatic detection. Journal of Vision, 17(13:9):1-18, doi:10.1167/17.13.9., 2017.

A. E. Silva and C. Chubb. The 3-dimensional, 4-channel model of human visual sensitivity to grayscale scrambles. Vision Research, 101:94 - 107, 2014.

C. F. Stromeyer, J. Lee, and R. T. Eskew. Peripheral chromatic sensitivity for flashes: a opost-receptoral red-green asymmetry. Vision Research, 32(10):1865-1873, 1992.

P. Sun, C. Chubb, C. E. Wright, and G. Sperling. Human attention filters for single colors. Proceedings of the National Academy of Sciences, USA, pages E6712-E6720, www.pnas.org/cgi/doi/10.1073/pnas.1614062113, 2016.

B. W. Tansley and R. M. Boynton. Chromatic border percetpion: the role of red- and green-sensitive cones. Vision Research, 18:683-697, 1978. 
B. W. Tansley and R. J. Glushko. Spectral sensitivity of long-wavelength-sensitive photoreceptors in dochromats determined by elimination of border percepts. Vision Research, 18: 699-706, 1978.

J. P. H. van Santen and G. Sperling. Temporal covariance model of human motion perception. Journal of the Optical Society of America A, 1:451-473, 1984.

J. P. H. van Santen and G. Sperling. Elaborated reichardt detectors. Journal of the Optical Society of America A, 2:300-321, 1985.

W. Verdon and A. J. Adams. Short-wavelength sensitive cones do not contribute to mesopic luminosity. Journal of the Optical Society of America A, 4:91-95, 1987.

J. D. Victor, M. M. Conte, and C. Chubb. Textures as probes of visual processing. Annual review of vision science, 3:275-296, 2017.

J. W. T. Walsh. Photometry. Constable and Company LTD, 1958.

A. B. Watson and A. J. Ahumada. A model of human visual-motion sensing. Journal of the Optical Society of America A, 2:322-342, 1985.

A. B. Watson and J. G. Robson. Discrimination at threshold: Labelled detectors in human vision. Vision Research, 21:1115-1122, 1981.

\section{APPENDIX}

\section{The Legendre polynomials}

The functions used in our experiments are discrete domain analogs of the Legendre polynomials (which are continuous functions on the interval $[-1,1]$ ). Let $\omega_{j}, j=1,2 \cdots, 8$, be the 8 colors (ranging from green to red) used in our stimulus scrambles. Then the functions we use are derived by applying Gram-Schmidt orthonormalization to the monomials $f_{k}\left(\omega_{j}\right)=j^{k}$, for $k=0,1, \cdots, 7$. The functions $\lambda_{1}, \lambda_{2}, \cdots, \lambda_{7}$ are given in table $2 . \quad\left(\lambda_{0}(j)=\frac{1}{\sqrt{8}}\right.$ for $j=1,2, \cdots, 8$; this function plays no role in our experiments.)

\begin{tabular}{c|cccccccc}
$k$ & $\lambda_{k}(1)$ & $\lambda_{k}(2)$ & $\lambda_{k}(3)$ & $\lambda_{k}(4)$ & $\lambda_{k}(5)$ & $\lambda_{k}(6)$ & $\lambda_{k}(7)$ & $\lambda_{k}(8)$ \\
\hline 1 & -0.5401 & -0.3858 & -0.2315 & -0.0772 & 0.0772 & 0.2315 & 0.3858 & 0.5401 \\
\hline 2 & 0.5401 & 0.0772 & -0.2315 & -0.3858 & -0.3858 & -0.2315 & 0.0772 & 0.5401 \\
\hline 3 & -0.4308 & 0.3077 & 0.4308 & 0.1846 & -0.1846 & -0.4308 & -0.3077 & 0.4308 \\
\hline 4 & 0.2820 & -0.5238 & -0.1209 & 0.3626 & 0.3626 & -0.1209 & -0.5238 & 0.2820 \\
\hline 5 & -0.1498 & 0.4922 & -0.3638 & -0.3210 & 0.3210 & 0.3638 & -0.4922 & 0.1498 \\
\hline 6 & 0.0615 & -0.3077 & 0.5539 & -0.3077 & -0.3077 & 0.5539 & -0.3077 & 0.0615 \\
\hline 7 & -0.0171 & 0.1195 & -0.3585 & 0.5974 & -0.5974 & 0.3585 & -0.1195 & 0.0171 \\
\hline
\end{tabular}

Table 2: The Legendre polynomials of order 1 to 7 . 


\section{Fitting the Descriptive and Tool-construction models.}

The details of the Bayesian method used to estimate the joint posterior density characterizing the parameters of each of the two models used in the paper are described in the Supplementary materials. The data from the experiment and the Matlab code used to fit all models are archived in Open Science Framework project, https://osf.io/tyg52/ (entitled, "Color scrambles reveal Red and Green half-wave linear mechanisms plus a mechanism selective for low chromatic contrast: Data and code for analysis") For each model, we use Markov chain Monte Carlo (McMC) simulation to derive a sample from the posterior density characterizing the model parameters. In order to use this procedure we need to specify the likelihood function for each model. This is the purpose of the following two sections.

\section{The likelihood function for the descriptive model}

The descriptive model allows all of the different tools $T_{s, t}$ achieved by different subjects $s$ in different tasks $t$ to be freely determined under the single constraint that subject $s$ uses the same Weibull steepness parameter $B_{s}$ in all four tasks. Thus, the descriptive model is fit separately for each subject. For a given subject $s$, the parameters are $T_{s, t}(\omega)$, for all $\omega \in \Omega$ and $t=1,2,3,4$. However, any given tool $T_{s, t}$ is constrained to sum to 0 . Thus, for a given subject this model has $4 \times 7+1=29$ free parameters; hence, in all, the model has $5 \times 29=145$ free parameters.

For a given subject $s$ and task $t$, let $C_{t}$ be the set of all trials in task $t$ on which the subject responded correctly, and let $I_{t}$ be the set on which he-or-she responded incorrectly, and let $\rho_{t, k}$ be the perturbation used to generate the stimulus on trial $k$ in task $t$. Then the likelihood function of the descriptive model for a given subject $s$ is:

$$
\Lambda_{s}=\prod_{t=1}^{4} \prod_{k \in C_{t}} \Psi_{s}\left(T_{s, t} \bullet \rho_{t, k}\right) \prod_{k \in I_{t}}\left(1-\Psi_{s}\left(T_{s, t} \bullet \rho_{t, k}\right)\right) .
$$

where $\Lambda_{s}$ is implicitly a function of the model parameters for subject $s$.

\section{The likelihood function for the tool-construction model}

\section{The parameters of the tool-construction model}

The tool-construction model is a nested version of the descriptive model in which the tool $T_{s, t}$ deployed by subject $s$ in task $t$ is constructed from a fixed set of mechanism sensitivity functions. We use McMC simulation to fit the tool-construction model for $M=2,3,4$ mechanisms. Each of mechanisms $m=1,2, \cdots, M$ is characterized by a function $F_{m}: \Omega \rightarrow$ $\mathbb{R}$. However, because we assume the sensitivity functions characterizing mechanisms $m$ have different amplitudes in different subjects, we can assume without loss of generality that the functions $F_{m}$ (which determine only the relative activations produced by the different colors

in $\Omega$ ) are normalized: $\sum_{\omega \in \Omega} F_{m}^{2}(\omega)=1$. In addition, our experimental paradigm only allows us to measure the projection of $F_{m}$ into the space of perturbations, each of which sums to 0 . This means that we can determine $F_{m}$ only up to the unknown additive constant that reflects the mean activation produced in mechanism $m$ by $\Omega$-scramble with histogram $U$. Thus, the 
$M$ functions $F_{m}$ add $M \times 6$ free parameters. In addition, the amplitudes $A_{s, m}$ of mechanisms $m=1,2 \cdots, M$ in all five subjects $s$ add $M \times 5$ free parameters. For each subject $s$ we also have a mechanism-combination parameter $\beta_{s}$ and Weibull function steepness parameter $B_{s}$ adding 10 more parameters. Thus, in all, the $M$-mechanism tool-construction model has $M \times(6+5)+10$ free parameters.

The likelihood function for the tool-construction model is given by Eq. 13 using tools $T_{s, t}$ given by Eq. 9 constructed using mechanism sensitivity functions $F_{s, m}$ given by Eq. 8 . The values $A_{s, m}$ in Eq. 8 are model parameters as are the values $F_{m}(\omega)$ for all $\omega \in \Omega$. Crucially, the weights $w_{s, t, m}$ used by subject $s$ to combine different mechanisms $m$ to produce the tool $T_{s, t}$ he-or-she uses in task $t$ are not free parameters. The assumption that these weights are chosen to optimize performance under the constraints of Eq. 10 determines these weights from the other model parameters. Thus, the values $w_{s, t, m}$ in Eq. 9 need to be derived from the model parameters. As we show in the next section, these weights are given by Eqs. 21 and 22 below.

\section{Deriving the weights $w_{s, t, m}$.}

In this section we focus on the case of a single subject $s$ performing a single task $t$. To simplify notation, we drop the subscripts $s$ and $t$ and refer to the weights $w_{s, t, m}$ as $w_{m}$.

Suppose the subject possesses mechanisms $m=1,2, \cdots, M$ with sensitivity functions $F_{m}$ (given by Eq. 8) and uses combination exponent $\beta$ to construct tools from these mechanisms. We assume that when the subject performs the task with seed perturbation $\phi$, he-or-she chooses the combination weights $w_{m}$ optimally under the constraint that

$$
\sum_{m=1}^{M} w_{m}^{\beta}=C
$$

for some arbitrary constant $C$ (that we will set to 1 ). In order to compute the likelihood function for the model that rests on these assumptions, we need a formula for the optimal weights $w_{m}$ given

1. the functions $F_{m}$,

2. the combination parameter $\beta$,

3. and the target perturbation $\phi$.

Specifically, we need a formula for the the weights $w_{m}$ maximizing $\operatorname{Tool}^{T} \phi$, where

$$
\text { Tool }=\sum_{m=1}^{M} w_{m} F_{m}
$$

under the constraint of Eq. 14.

We can always start by setting to 0 all $w_{m}$ for which $F_{m}^{T} \phi \leq 0$. This lets us ignore the terms $m$ in the sum for which $F_{m}^{T} \phi \leq 0$; we thus assume without loss of generality that $F_{m}^{T} \phi>0$ for all $m$ in the sum. Let $F$ be the matrix whose column vectors are the sensitivity 
functions $F_{m}, m=1,2 \cdots, M$, and let $w$ be the column vector with values $w_{1}, w_{2}, \cdots, w_{M}$. Then

$$
\text { Tool }=F w
$$

and our aim is to maximize

$$
[F w]^{T} \phi=w^{T} V \text { for } \quad V=F^{T} \phi
$$

We approach the problem by introducing a Lagrange multiplier $L$ as follows: Define the function

$$
\Psi(w, L)=w^{T} V+L\left(\sum_{m=1}^{M} w_{m}^{\beta}-C\right),
$$

and note that the values of $w_{m}$ obtained at a local maximum of $\Psi$ will satisfy the target conditions. The partial derivatives of $\Psi$ with respect to the $w_{m}$ 's and $L$ are

$$
\frac{\partial \Psi}{\partial w_{m}}=V_{m}+L \beta w_{m}^{\beta-1} \quad m=1, \cdots, M
$$

and

$$
\frac{\partial \Psi}{\partial L}=\sum_{m=1}^{M} w_{m}^{\beta}-C .
$$

By design, setting Eq. 20 to 0 yields Eq. 14. More interestingly, setting Eq. 19 to 0 yields

$$
w_{m}=\gamma \tilde{w}_{m} \quad \text { for } \quad \tilde{w}_{m}=V_{m}^{\frac{1}{\beta-1}}
$$

where $\gamma=(L \beta)^{-\frac{1}{\beta-1}}$. Equation 21 determines the relative values of the weights $w_{m}$. These are the values $\tilde{w}_{m}$. Variations in the amplitude $\gamma$ (which can be produced by varying $L$ ) influence only the value of $C$ in Eq. 14 .

We make the $w_{m}$ 's satisfy Eq. 14 with $C=1$ by setting

$$
\gamma=\left(\sum_{k=1}^{n} \tilde{w}_{k}^{\beta}\right)^{-\frac{1}{\beta}}
$$

